\title{
Solidarität mit Geflüchteten und Fallstricke des Helfens
}

\author{
Silke van Dyk • Laura Boemke • Tine Haubner
}

Angenommen: 25. Oktober 2021 / Online publiziert: 22. November 2021

(C) Der/die Autor(en) 2021

Zusammenfassung Der Beitrag untersucht am Beispiel des Engagements für Geflüchtete Stärken, Herausforderungen und Probleme freiwilligen Engagements und arbeitet zugleich die Spezifika dieser Form der Hilfe und Solidarität heraus. Die Analyse rekurriert auf eine qualitative Erhebung, die problemzentrierte Interviews mit Engagierten und Leitfadeninterviews mit Expert*innen sowie eine Dokumentenanalyse von politischen, medialen, zivilgesellschaftlichen und wissenschaftlichen Quellen für den Zeitraum von 2011 bis 2018 umfasst. Im Zentrum der Analyse stehen (1) der mediale und gesellschaftliche Außenblick auf die ehrenamtliche Flüchtlingshilfe, (2) multiple Grenzziehungen, -überschreitungen und -erfahrungen im Engagement sowie (3) die Bewältigung von Problemen und Herausforderungen seitens der Engagierten - durch den Ausstieg aus dem Engagement, die Kritik an Engagementbedingungen, die Verheimlichung des Engagements oder die Politisierung der Rolle freiwilliger Hilfe im Strukturwandel des Wohlfahrtsstaats.

Schlüsselwörter Flüchtlingshilfe · Ehrenamtliches Engagement · Solidarität · Rassismus · Willkommenskultur

Silke van Dyk $(\bowtie) \cdot$ Laura Boemke $\cdot$ Tine Haubner Institut für Soziologie, Friedrich-Schiller-Universität Jena, Carl-Zeiß-Str. 3, 07743 Jena, Deutschland E-Mail: silke.vandyk@uni-jena.de

Laura Boemke

E-Mail: laura.boemke@uni-jena.de

Tine Haubner

E-Mail: tine.haubner@uni-jena.de 


\title{
Solidarity with refugees and the pitfalls of helping
}

\begin{abstract}
The paper turns to the example of voluntary assistance of refugees to explore the strong points, challenges, and problems of voluntary aid, in an attempt to map out the specifics of this form of assistance and solidarity. The analysis is based on a qualitative study which comprises problem centered interviews with volunteers and guided interviews with experts as well as a document analysis of sources from the spheres of politics, the media, civil society and science dating from 2011 to 2018. The investigation focuses (1) on the outside view on voluntary refugee aid articulated in the media and society at large, (2) on the multiple demarcations, transgressions and experience of limits in volunteering, and (3) on the ways in which volunteers deal with problems and challenges-by quitting volunteering, by criticizing the conditions of their commitment or by hiding it, or by politicizing the role voluntary assistance plays in the restructuring of the welfare state.
\end{abstract}

Keywords Refugee assistance $\cdot$ Voluntary work $\cdot$ Solidarity $\cdot$ Racism $\cdot$ Welcome Culture

\section{La solidarité avec les réfugiés et les écueils de l'aide}

Résumé Cet article étudie les forces, les défis et les problèmes de l'engagement bénévole à partir de l'exemple de l'engagement en faveur des réfugiés tout en mettant en évidence les spécificités de cette forme d'aide et de solidarité. L'analyse est basée sur une enquête qualitative comprenant des entretiens centrés sur un problème avec des personnes engagées, des entretiens semi-directifs avec des experts et des expertes ainsi qu'une analyse de documents de sources diverses (politique, médias, société civile, recherche scientifique) sur une période allant de 2011 à 2018. L'analyse est axée sur (1) la perception médiatique et sociale de l'aide bénévole aux réfugiés, (2) les multiples démarcations, transgressions et expériences limites dans le cadre de l'engagement ainsi que (3) la gestion des problèmes et des défis par les personnes engagées - par le désengagement, la critique des conditions d'engagement, la dissimilation de l'engagement ou la politisation du rôle de l'aide bénévole sur fond de transformation structurelle de l'État-providence.

Mots-clés Aide aux réfugiés · Engagement bénévole · Solidarité · Racisme Culture de l'accueil

\section{Einleitung}

Der „lange Sommer der Migration“ (Hess et al. 2017), die „Willkommenskultur“ und das große Ausmaß der ehrenamtlichen Hilfe sind seit 2015 politisch, medial und wissenschaftlich viel diskutiert worden (vgl. im Überblick: van Dyk 2019). Die Flüchtlingshilfe galt vielen als Sternstunde der deutschen Zivilgesellschaft, als leuchtendes Beispiel des „guten“ Deutschland, das gegen Angriffe auf Geflüchtete und Helfer*innen hochgehalten wird. Und es dauerte nicht lange, bis der selbsterklärte 
„Aufarbeitungsweltmeister“ seiner NS-Vergangenheit als „das neue Deutschland“ (Amann et al. 2015) in der medialen Darstellung zum Weltmeister der Willkommenskultur avancierte. Tatsächlich zeigen verschiedene Untersuchungen und Schätzungen, dass die Unterstützung - zumal in den ersten Monaten - sehr groß war, gehen doch selbst moderate Schätzungen davon aus, dass sich ca. zehn Prozent der erwachsenen Bevölkerung aktiv (mit Zeitspenden) engagiert haben - eine Quote, die noch beträchtlich steigt, wenn zusätzlich Sach- und Geldspenden Berücksichtigung finden (BMFSFJ 2017, S. 8 f.). Diese umfangreiche Unterstützung verdankte sich auch positiver Resonanz seitens staatlicher Behörden und medialer Berichterstattung: „The positive response on the part of German authorities and the media helped to turn a pre-existent but small volunteer movement into a mainstream initiative, involving large and diverse parts of German society“ (Karakayali 2017, S. 8).

Die große Aufmerksamkeit für die ehrenamtliche Flüchtlingshilfe in Verbindung mit der unbestrittenen Notwendigkeit der Nothilfe hat zugleich dazu beigetragen, grundsätzliche Probleme und Herausforderungen freiwilligen Engagements in die öffentliche Debatte zu holen: Die Instrumentalisierung Freiwilliger für die Gewährleistung existenzieller Daseinsvorsorge hat ebenso Kritik auf sich gezogen wie die Überforderung vieler Engagierter; erstmals wurde in einer breiteren Öffentlichkeit diskutiert, ob und wo unbezahlte Engagierte und Laien genuin professionelle Aufgaben übernehmen oder dafür sorgen, dass Menschen ihre staatlich verbürgten Rechte überhaupt wahrnehmen können. Im wissenschaftlichen Feld sind in den Folgejahren nach 2015 eine Reihe kleinerer qualitativer Studien und wenige standardisierte Erhebungen zum Engagement für Geflüchtete erschienen, oftmals handelte es sich jedoch um Projekte mit eher kurzer Laufzeit (z.B. Bertelsmann-Stiftung 2016; Gesemann und Roth 2017; Vey und Sauer 2016; Hamann et al. 2016; BMFSFJ 2017; Speth und Becker 2016; Beckmann et al. 2018; vgl. im Überblick: Kleist 2018, S. 30f.). Gerade die langfristigen Herausforderungen der Umstellung von der Nothilfe unter Ausnahmebedingungen auf die Verstetigung und Professionalisierung der ehrenamtlichen Unterstützung bleiben deshalb bislang unterbelichtet, obwohl sich Struktur und Inhalt der von Engagierten übernommenen Aufgaben stark gewandelt haben: „Die erste Phase der Hilfe war die Stunde des Bürgerschaftlichen Engagements [...]. Sie war geprägt durch Tempo, Improvisation, Spontaneität. [...] In der jetzt angelaufenen zweiten Phase befinden wir uns nicht mehr im Katastrophenmodus“ (Zeckra 2017, S. 1).

Auf Basis einer eigenen qualitativen Erhebung, die problemzentrierte Interviews mit Engagierten, Expert*inneninterviews, eine kleine Zahl an Gruppendiskussionen und eine Dokumentenanalyse umfasst (vgl. Abschnitt 2), nutzen wir die Flüchtlingshilfe als Brennglas für Stärken, Herausforderungen und Probleme freiwilligen Engagements und fragen zugleich nach den Spezifika dieser Form der Hilfe und Solidarität. ${ }^{1}$ Wir analysieren, inwiefern das Engagement für Geflüchtete nicht nur besondere Aufmerksamkeit und Anerkennung auf sich zieht, sondern auch - und im Gegensatz zu anderen Engagementfeldern - Angriffe und Kritik, die an die Enga-

\footnotetext{
${ }^{1}$ Eine Kurzfassung dieser Analyse, die sich ausschließlich auf das Interviewmaterial stützt, ist in einer Publikation der Landeszentrale für politische Bildung Baden-Württemberg erschienen (van Dyk et al. 2020).
} 
gierten besondere Anforderungen stellen. Um der Frage nachzugehen, ob sich die ehrenamtliche Flüchtlingshilfe als die „Sternstunde der Zivilgesellschaft“ erweist, als die sie gefeiert worden ist, ist es zudem erforderlich, insbesondere in Bezug auf die Handlungsbedingungen freiwilliger Hilfe auch Probleme und Schwachstellen systematisch zu analysieren und eine Einbettung des Untersuchungsgegenstandes in die übergreifende Entwicklung der „Verzivilgesellschaftlichung der sozialen Frage" vorzunehmen. Diese Entwicklung zielt, wie wir an anderer Stelle ausführlich gezeigt haben (van Dyk und Haubner 2019; Boemke et al. 2021), auf die Aktivierung nicht-erwerbsförmiger Sorgetätigkeiten im Rahmen einer sozialpolitischen Gabenökonomie, in Gestalt unbezahlter oder geringfügig entschädigter freiwilliger Tätigkeiten jenseits von Staat, Markt und Familie. Vor diesem Hintergrund vertreten wir die These, dass die freiwillige Nothilfe 2015 und 2016 nicht nur wesentlich dazu beigetragen hat, die außergewöhnliche Situation trotz staatlicher Untätigkeit zu bewältigen, sondern dass in diesem Prozess Grenzen überschritten wurden, die das freiwillige Engagement im Regelfall fachlich, professionell sowie hinsichtlich seiner Regulierung vom qualifizierten Hauptamt und der Erwerbsarbeit unterscheiden. Damit sind sowohl die Überschreitung persönlicher Belastungsgrenzen von Engagierten als auch Grenzüberschreitungen im Umgang mit Geflüchteten gemeint, die nicht selten paternalistische Züge trugen. Nicht zuletzt sind politisch motivierte Grenzüberschreitungen gegenüber dem als repressiv kritisierten Staat und einer flüchtlingsfeindlichen oder intransparenten Bürokratie von zentraler Bedeutung.

Die folgende Analyse verfährt in drei Schritten und untersucht im Anschluss an kurze Hinweise zur empirischen Basis und methodischen Vorgehensweise (Abschnitt 2) den medialen und gesellschaftlichen Außenblick sowie soziale Reaktionen auf die ehrenamtliche Flüchtlingshilfe (Abschnitt 3), die multiplen Grenzziehungen, -überschreitungen und -erfahrungen in der freiwilligen Flüchtlingshilfe (Abschnitt 4) sowie die Bewältigung von Problemen und Herausforderungen durch die Engagierten selbst - durch den Ausstieg aus dem Engagement, die Kritik an Engagementbedingungen, die Verheimlichung des Engagements oder die Politisierung der Rolle freiwilliger Hilfe im Strukturwandel des Wohlfahrtsstaats (Abschnitt 5). Für die Systematisierung der Bewältigungsweisen greifen wir auf die von Albert Hirschman (1970) entwickelte begriffliche Trias aus Exit, Voice und Loyality zurïck.

\section{Empirische Basis und methodisches Vorgehen}

Der Beitrag basiert auf dem von der Hans-Böckler-Stiftung geförderten Forschungsprojekt ,Schattenökonomie oder neue Kultur des Helfens? Engagement und Freiwilligenarbeit im Strukturwandel des Wohlfahrtsstaats“, das von 2017 bis 2020 am Institut für Soziologie der Universität Jena angesiedelt war. Die Ergebnisse zur Flüchtlingshilfe, die diesem Beitrag zugrunde liegen, gehen auf die Auswertung von 14 Expert*innengesprächen, neun problemzentrierten Interviews und zwei Gruppendiskussionen mit Engagierten in der Flüchtlingshilfe sowie vier teilnehmenden Beobachtungen bei Schulungs- und Supervisionsveranstaltungen zurück. Darüber hinaus greifen wir auf Aspekte aus 21 Interviews mit Engagierten und 31 Interviews mit hauptamtlichen Expert*innen zurück, die in anderen Feldern engagiert bzw. 
einschlägig sind, sich aber ebenfalls - teilweise ausführlich - zur Flüchtlingshilfe äußern. Erhoben wurde 2018 und 2019 in Berlin, Brandenburg und Baden-Württemberg, die konkreten Untersuchungsorte in Brandenburg und Baden-Württemberg wurden ebenso wie die Interviewpartner*innen anonymisiert.

Um den Prozess der Interviewauswertung zu systematisieren, haben wir bei den problemzentrierten Interviews mit Engagierten Anleihen bei der Dokumentarischen Methode genommen, die im Anschluss an die Wissenssoziologie Karl Mannheims darauf zielt, den impliziten Sinngehalt von Interviewtexten zu rekonstruieren (Bohnsack 2003). Dem liegt die Annahme zugrunde, dass eine Analyse über den wörtlichen und expliziten Gehalt des Gesagten - den intentionalen Ausdruckssinn - hinausgehen und die Aufmerksamkeit auf die implizite Sinnstruktur - den Dokumentsinn richten muss, die die im Interview verstreuten Äußerungen zusammenhält. Im Vergleich der Fälle untereinander galt unser Interesse der Frage, inwiefern vor dem Hintergrund unterschiedlicher Orientierungsrahmen der Interviewten ähnliche Themen (z.B. die eigene Überlastung, die Zusammenarbeit mit Hauptamtlichen oder die Kritik an Behörden) verhandelt werden. Die Orientierungsrahmen haben wir ausgehend von der Trias „Engagementverständnis“, „Gesellschaftsbild“, „Staatsverständnis/Rolle des Sozialstaats“ herausgearbeitet, wodurch eine Kontextualisierung des intentionalen Ausdruckssinns und die Analyse des Dokumentsinns ermöglicht wurde. Auch die Gruppendiskussionen haben wir nach Maßgabe der Dokumentarischen Methode analysiert; mit diesem Format ging es uns darum, Interaktionen und Kontroversen unter Engagierten in ihrer Eigendynamik einzufangen. Die Expert*inneninterviews wurden anhand eines thematischen Leitfadens ausgewertet, der die zentralen Forschungsthesen des Projekts - ,Instrumentalisierung“, „Informalisierung“, „De-Professionalisierung“ und „Politisierung“ von Engagement - zum Ausgangspunkt nimmt und um zentrale Aspekte ergänzt, die für die jeweilige Expertise relevant sind.

Der Korpus der Dokumentenanalyse umfasst 1250 Dokumente von politischen Parteien, Regierungsvertreter*innen und Abgeordneten, Kirchen, Gewerkschaften, Wohlfahrtsverbänden, Freiwilligenorganisationen und Wissenschaftler*innen sowie einschlägige Ratgeber und Beiträge aus der regionalen und überregionalen Presse (die tageszeitung, Frankfurter Allgemeine Zeitung, SPIEGEL, Süddeutsche Zeitung, Stuttgarter Zeitung, Lokalzeitung der baden-württembergischen Stadt C, Märkische Allgemeine). Mit dem Korpus erschließen wir systematisch und auf der Basis heterogener Quellen die Debatten und einzelnen Positionierungen zur Flüchtlingshilfe über mehrere Jahre hinweg, um sowohl deren ungemeine Popularität und öffentliche Präsenz seit dem Sommer 2015 als auch die Interviews mit Engagierten und Expert*innen in einen empirisch fundierten Kontext einordnen zu können. Die mit MAXQDA erfolgte thematische Kodierung orientierte sich am Kodierparadigma der Grounded Theory, ohne dass die Schritte des offenen, axialen und selektiven Kodierens dabei vollständig übernommen wurden: Sie stellt vielmehr eine Mischform des offenen und axialen Kodierens dar, insofern sie über eine theoretisch weitgehend offene, sequenzanalytische Konzeptentwicklung hinausgeht, ohne den Fokus bereits auf die Analyse einzelner Konzepte zu verengen. Die thematischen Codes wurden auf Basis der sekundäranalytischen und theoretischen Sensibilisierung für konkrete engagementbezogene Konzepte entwickelt und im Sinne einer In-vivo-Kodierung im 
Kodierprozess selbst permanent erweitert und modifiziert (vgl. zu dieser Vorgehensweise auch: Denninger et al. 2014, S. 54 ff.). Im Sinne der ,,aufsteigenden Analyse“ der Grounded-Theory-Methodologie haben wir konzept- und theoriegenerierend gearbeitet (Strauß 2004), ohne deshalb auf eine Systematisierung der Vorannahmen zu verzichten.

\section{Flüchtlingshilfe: „Hip“ und anerkannt oder blockiert und bedroht?}

Im Sommer 2015 entstand eine Welle und Bewegung des Helfens, die mobilisierend wirkte und die Flüchtlingshilfe vorübergehend zu einem ,place to be“ werden ließ, der zugleich Anerkennung und Aufmerksamkeit versprach. Diese Anziehungskraft blieb - zumal in den größeren Städten - geraume Zeit erhalten, wie es ein in der Rechtsberatung Engagierter im „Migration Hub“, einem Treffpunkt unterschiedlicher Initiativen in Berlin, auf den Punkt bringt: „Das Flüchtlingsthema [...], es ist gerade hip. Viele Leute möchten sich im Flüchtlingsbereich engagieren. Ist einfach attraktiv." (Martin Kaiser, Z. $232 \mathrm{ff.}$ )

Demgegenüber beklagen Interviewte aus anderen Engagementbereichen ${ }^{2}$, dass die Flüchtlingshilfe finanzielle und personelle Ressourcen abgezogen habe, die vorhandenen Nachwuchsprobleme im Ehrenamt verschärfe und zudem ein Maß an Anerkennung verspreche, von dem andere Engagierte nur träumen könnten - wie z.B. ein für die Fachberatung Engagierter in Baden-Württemberg zuständiger Experte betont: „Das ist eher das Problem, dass alle nur noch Flüchtlingshilfe machen wollen und nicht mehr in den Altenheimen oder was weiß ich. [...] Die wollen was erleben, wollen eine Anerkennung.“ (Herbert Zillmann, Z. $360 \mathrm{ff}$.) Mit ähnlichem Tenor moniert eine Vertreterin der Caritas: „Die Flüchtlinge haben auch sehr viel Ehrenamtskapazität abgezogen. Die haben eine Lobby und das ist toll. Zur mir hat gerade jemand gesagt: Es ist gerade schick, zu den Flüchtlingen zu gehen. Das steht dann in der Zeitung, da wird man anerkannt. Wenn man sagt, zu den Alten, dann höre ich: ,Du und deine Alten. ““ (Ilse Schlegel, Z. 552 ff.) Empirisch deutet bislang jedoch wenig darauf hin, dass Engagierte bei der Feuerwehr oder in der schulischen Nachmittagsbetreuung plötzlich in die Flüchtlingshilfe gewechselt sind; vielmehr wurden vormals nicht Engagierte im ,langen Sommer der Migration“ mobilisiert, bereits Engagierte haben sich zusätzlich für Geflüchtete eingesetzt (BMFSFJ 2017), und die Flüchtlingsthematik wurde als neues Querschnittsthema in vielen Bereichen - im Sport, in der Bildung oder in Mehrgenerationenprojekten - verankert. ${ }^{3}$ In der politischen Engagementförderung ist dabei durchaus eine starke Konzentration

\footnotetext{
2 Hier erweist es sich als Vorteil, dass unsere Untersuchung auch Perspektiven auf die Flüchtlingshilfe von Engagierten und Expert*innen aus anderen Bereichen erhoben hat - bislang ein Alleinstellungsmerkmal in qualitativen Studien zur Unterstützung von Geflüchteten.

3 So bieten im Rahmen des 2017 neu aufgelegten Bundesprogramms „Mehrgenerationenhaus“ 490 der 550 geförderten Häuser den fakultativen Schwerpunkt „Integration von Menschen mit Migrations- und Fluchtgeschichte“ an. Eine Umfrage der Bundesarbeitsgemeinschaft der Freiwilligenagenturen hat ergeben, dass alle befragten Agenturen in der Flüchtlingshilfe aktiv sind (bagfa 2016). Zu nennen ist ferner das Programm „Bundesfreiwilligendienst mit Flüchtlingsbezug“, das zusätzlich zu den bestehenden (jährlich) 37.000 Plätzen für einen Zeitraum von drei Jahren 10.000 neue Stellen geschaffen hat (BAFzA 2015).
} 
auf das Engagementfeld Flüchtlingshilfe zu konstatieren: So weist eine Interviewpartnerin aus dem Ministerium für Soziales und Integration in Baden-Württemberg darauf hin, dass die Landesengagementstrategie gänzlich auf das Thema der Integration ausgerichtet worden sei, was in verschiedenen Kontexten für Unfrieden gesorgt habe.

Zugleich lassen mehrere der von uns interviewten Engagierten erkennen, dass es ihnen nicht nur um ein vermeintliches Übermaß an Aufmerksamkeit für die Flüchtlingshilfe geht, sondern dass dem auch Vorstellungen darüber zugrunde liegen, wem Hilfe ,eigentlich“ gebühren würde. Dies ist beispielsweise der Fall, wenn die Vertreterin der Caritas die von ihr als vernachlässigt beklagten Alten selbstverständlich als „deutsche Senior*innen“ bezeichnet. Ähnlich antwortet eine Engagierte beim Kinderschutzbund im baden-württembergischen D auf die Frage, ob die Anerkennung von Engagement zugenommen habe: „Es hat zugenommen, eben wie gesagt durch die Flüchtlinge, da haben sich sehr viele überlegt [...] , mir geht es gut, ich kann da helfen'. Das schon. Aber die richtigen Armen, also jetzt unsere Armen, nein.“ (Henriette Olcher, Z. $758 \mathrm{ff}$.) Eine solche Vorstellung exklusiver Solidarität im Sinne einer legitimen Anwartschaft bestimmter Bevölkerungsgruppen findet sich auch in zwei Gruppendiskussionen mit Engagierten wieder, die nicht schwerpunktmäßig in der Flüchtlingshilfe aktiv sind: Eine Engagierte der Gruppe „Freizeitengel“ im baden-württembergischen $\mathrm{C}$, die für mobilitätseingeschränkte ältere Menschen Freizeitaktivitäten und Alltagsversorgung organisieren, bekundet großes Verständnis für den im Februar 2018 erklärten Aufnahmestopp für Nichtdeutsche bei der Essener Tafel: ,Ja, das ist ja das Thema in Essen, dass die gesagt haben, die verdrängen die eigentlich Bedürftigen, [...] dass man sagt ,Ja, die muss man ausgrenzen, weil sonst für die, die sonst auch immer kommen, nichts mehr übrig bleibt'." (Gruppendiskussion Freizeitengel, Z. 26 ff.) Ähnlich äußern sich Engagierte des Tafelladens im baden-württembergischen C (Gruppendiskussion Tafelladen in C, Z. 35 f.).

Interessanterweise findet sich die Annahme von einer hochgelobten und breit geförderten Flüchtlingshilfe vornehmlich bei denjenigen, die nicht in diesem Feld aktiv sind. Aus der Binnensicht hingegen zeigt sich, standardisierten Befragungen von Engagierten in der Flüchtlingshilfe zufolge, ein anderes Bild (vgl. z.B. Ministerium für Arbeit, Soziales, Gesundheit, Frauen und Familie Brandenburg 2018, S. 56f.; Hamann et al. 2016). Auch viele der von uns Interviewten kritisieren die mangelhafte und zu kurzfristige Förderung einzelner Hilfsprojekte („Projektitis“), Blockaden durch kommunale Akteure, Probleme mit Behörden und soziale Ächtung bis hin zu rassistisch motivierten Angriffen auf Engagierte und Geflüchtete. Flüchtlingshelfer*innen würden, so ein Engagierter des Unterstützerkreises Asyl im badenwürttembergischen C, von den Behörden als ,notwendiges Übel“ (Rolf Thoma, Z. 578) angesehen und ausgebremst. Auch mehrere Engagierte im brandenburgischen Landkreis B sehen in den vielen ,,bürokratischen Hürden“ (Lena Jung, Z. 571) eine mangelnde Wertschätzung ihres Einsatzes. ${ }^{4}$ In einem offenen Brief von 100 flüchtlingspolitischen Initiativen im Bundesland Brandenburg heißt es: „In vielen Fällen übernehmen wir unentgeltlich Beratungs- und Versorgungsaufgaben, die eigentlich

\footnotetext{
${ }^{4}$ Es gibt im Sample auch Beispiele für eine sehr enge, als fruchtbar erlebte Kooperation von Engagierten und Behörden, allerdings scheint dies eher seltener der Fall zu sein.
} 
staatliche Aufgaben wären. Allerdings scheint in vielen Fällen unsere Arbeit nicht ,willkommen` zu sein. Allzu häufig haben wir das Gefühl uns zunächst gegen unsinniges Verwaltungshandeln durchsetzen zu müssen." (Offener Brief flüchtlingspolitischer Initiativen in Brandenburg 2015) Diese Kritik wiegt umso schwerer, als die am häufigsten erbrachte Hilfeleistung in der Flüchtlingshilfe die Unterstützung von Geflüchteten bei Behördengängen und Anträgen ist (Karakayali und Kleist 2015, S. 28; Ministerium für Arbeit, Soziales, Gesundheit, Frauen und Familie Brandenburg 2018, S. 20). Was die soziale Anerkennung betrifft, kommt hinzu, dass das Engagement in der Flüchtlingshilfe, aufgrund verbreiteter flüchtlingsfeindlicher Positionen in der Gesellschaft, grundsätzlich kritischer bewertet wird als der Einsatz in anderen Engagementfeldern. Während die ehrenamtliche Unterstützung älterer Menschen in einer Online-Befragung im Ruhrgebiet nur von 2\% der Befragten abgelehnt wird, lehnen 19\% der Befragten das Engagement in der Flüchtlingshilfe ab. Auch ambivalente Einstellungen sind in Bezug auf die Flüchtlingshilfe häufiger, während der Einsatz für Ältere von $86 \%$ der Befragten als durchweg positiv gesehen wird (Beckmann et al. 2018, S. 31). Wie diese Daten zeigen, scheint sich „die ablehnende Haltung gegenüber Flüchtlingen“ in der Bewertung des entsprechenden Engagements widerzuspiegeln (ebd., S. 37), weshalb Beckmann et al. für die Flüchtlingshilfe von einer „Ablehnung zweiten Grades“ (ebd.) sprechen.

In unserer Untersuchung zeigt sich diesbezüglich ein starkes Ost-West- und StadtLand-Gefälle: Insbesondere im ländlichen, klein- und mittelstädtischen Brandenburg kann von einer großen, flächendeckenden Wertschätzung der Flüchtlingshilfe nicht die Rede sein; das behaupten hier - anders als in den baden-württembergischen Untersuchungsstädten - noch nicht einmal die Engagierten und Expert*innen aus anderen Feldern. ${ }^{5}$ Auch der „Hippness-Faktor“ aus Berlin scheint weiter entfernt zu sein, als es die wenigen Kilometer vermuten lassen würden. Engagierte und Hauptamtliche sind stattdessen mit negativen Reaktionen aus dem Arbeits-, Wohnund Familienumfeld konfrontiert, zuweilen auch mit kommunalen Akteuren, die das Engagement blockieren, sowie mit organisierten rechten Strukturen. Die professionell mit der Flüchtlingshilfe befasste Pfarrerin im brandenburgischen Landkreis B bemerkt: „Also, das erleben sie schon fast alle, dass es bekannt ist, dass sie hier sich engagieren in der Flüchtlingshilfe, und das eben ist natürlich auch, also es gibt Familien, die haben gesagt, die haben jetzt verabredet, dass über dieses Thema einfach bei Familienfeiern, Partys nicht mehr gesprochen wird.“ (Julia Rau, Z. 317 ff.) Bezüglich des Engagements in der Flüchtlingshilfe spricht sie von einer ,zerrissenen Stadt“ (Z. 301), und die stellvertretende Landrätin räumt ein: „Also wir haben Angriffe auf Flüchtlinge, wir haben sicherlich auch Beschimpfungen, wir haben bestimmt auch so eine Kneipen-Diskussion.“ (Jutta Keit, Z. 383 f.)

\footnotetext{
${ }^{5}$ Dieser Befund sollte nicht zu ausschließlich als Ost-West-Befund gelesen werden, zeigt die überregionale Presseberichterstattung doch, dass es in ländlichen Räumen in den alten Bundesländern zum Teil durchaus ähnliche Probleme geben kann (vgl. exemplarisch Kelnberger 2015). Qualitative Interviews mit Engagierten im Ruhrgebiet zeichnen jedoch ein gänzlich anderes Bild als unsere Erhebung in Brandenburg: Beckmann et al. (2018, S. 43) halten fest, dass ihre Befunde keine Hinweise darauf liefern, ,dass Engagierte in der Flüchtlingshilfe mehrheitlich mit massiven Widerständen in ihrem persönlichen Umfeld zu kämpfen haben“, betonen allerdings, dass es durchaus ambivalente Reaktionen gäbe.
} 
Die Sprecherin eines Aktionsbündnisses gegen Rassismus und Diskriminierung in Brandenburg weist ebenfalls auf Angriffe auf Engagierte durch Rechtsextreme hin und verwendet für das Engagement sogar den Begriff des „Outings“: ,Viele haben sich ja an ihrem Arbeitsplatz, in ihrer Familie, in der Kommune sozusagen nicht geoutet, dass sie Flüchtlinge unterstützen." (Hanna Zange, Z. 155ff.) Auch in der brandenburgischen Lokalpresse ist diese „,dunkle Seite der Zivilgesellschaft“ (Roth 2003) wiederkehrendes Thema - ganz anders als in Baden-Württemberg (Anonym 2015). Das Engagement ist hier keine an Ressourcen und Unterstützung reiche Anerkennungsquelle, sondern erfordert nicht selten ein starkes politisches Rückgrat. Das kann sowohl zu einer Politisierung ursprünglich karitativer Hilfe im Sinne eines dezidiert antirassistischen Engagements als auch zum sozialen Rückzug ${ }^{6}$ oder gar zur Verheimlichung des Engagements vor Familie, Kolleg*innen und Bekannten führen, wie auch andere Autor*innen betonen: „Aus Angst, vor Ort ausgeschlossen oder gar bedroht zu werden, entscheiden sich mitunter potenzielle Freiwillige gegen ein Engagement als Patin oder Pate oder engagieren sich verdeckt in so genannten geheimen Patenschaften." (Buscham und Gillwald 2018, S. 2; vgl. auch Abschnitt 4) Gegenüber diesem ,heimlichen Engagement“ sei sichtbares Engagement in der Flüchtlingshilfe, so eine Engagierte in Brandenburg, automatisch politisches Engagement mit einer klaren Positionierung: „Man muss es ja gar nicht sagen, aber mit dieser Tätigkeit [wird] ja eine persönliche Einstellung nach außen einfach bekundet. Ja? Ist klar, wenn ich in der Flüchtlingshilfe bin [...], dann ist das ja ein Statement." (Helene Kolb, Z. 65 ff.)

\section{Indienstnahme von Engagement und multiple Grenzüberschreitungen}

\subsection{Die Indienstnahme von Engagement und die Verzivilgesellschaftlichung der sozialen Frage}

Also auch jetzt die ganze Flüchtlingsproblematik. Also ich denke, ohne Ehrenamt wäre ja Deutschland zusammengebrochen. (Hannelore Rieflin, Engagierte im baden-württembergischen C, Z. 485 f.)

Nicht nur Engagierte empfinden das ehrenamtliche Engagement als unverzichtbar, selbst in einer Beschlussfassung des Bundestages heißt es, die ehrenamtliche Flüchtlingshilfe sei ,nicht länger als eine bloße Zutat zu verstehen, sondern als ein Schlüsselfaktor bei der Bewältigung der dringlichsten Aufgaben von heute“ (BMFSFJ 2016, S. 178). In politischen, medialen, kirchlichen, wohlfahrtsverbandlichen und wissenschaftlichen Verlautbarungen herrscht weitgehende Einigkeit dahingehend, dass das Engagement in der Hochphase des Flüchtlingszuzugs ein Rettungsanker in Zeiten staatlicher Überlastung war; Unterschiede treten hingegen in

\footnotetext{
6 So berichtet eine Ehrenamtskoordinatorin im brandenburgischen Landkreis B, dass eine Patin, die einen regelmäßigen Spielenachmittag in einem Flüchtlingsheim organisieren sollte, das Engagement mit der Begründung absagte, dass ,sie Angst hat, dass ihr Auto hier gesehen wird, und sie wohnen in Z, und dass dann die Leute ihr nicht wohlgesonnen sind. Also die Sorge war schon da." (Nadine Arndtner, Z. 844 ff.).
} 
der Bewertung dieses Umstands zutage. Die einen sehen in dieser Entwicklung vor allem die Stärke der Zivilgesellschaft und das Wunder der Alltagssolidarität (exemplarisch z.B. Kleist 2017; Phineo 2016; Joffe 2015)7, wobei selbst die Erkenntnis, dass das freiwillige Engagement notwendig ist, um genuinen staatlichen Verpflichtungen nachzukommen, nicht notwendigerweise als Problem erachtet wird: „Ohne das wunderbar große Potenzial ehrenamtlich und sozial Engagierter könnten die Kommunen, die Landkreise und die Landesregierung die gegenwärtig große Zahl von Menschen auf der Flucht kaum würdig unterbringen und betreuen. Und wir alle könnten dann weder dem Grundgesetz noch der Genfer Flüchtlingskonvention nachkommen." (Staatsministerium Baden-Württemberg 2015, S. 3)

Neben solch enthusiastischen Einschätzungen einer aufblühenden Zivilgesellschaft haben auch kritische Analysen an Gewicht gewonnen, die - so in Verlautbarungen von Oppositionsparteien, Wohlfahrtsverbänden, Wissenschaftler*innen sowie Teilen der regionalen und überregionalen Presse - vor allem das Staatsversagen problematisieren und die Kehrseiten dieser Entwicklung beleuchten. Thematisiert werden die Überlastung und Überforderung von Engagierten (z.B. Deggerich et al. 2015; Breining 2015), die staatliche Instrumentalisierung unbezahlter Arbeit, die Lücken der Daseinsvorsorge schließt (z.B. van Dyk 2019; Graf 2016; Wagenknecht 2016), sowie die Tendenzen der De-Professionalisierung, wenn Laien Aufgaben übernehmen, für die sie nicht qualifiziert sind - von Muttersprachler*innen ohne (pädagogische) Ausbildung, die Deutsch unterrichten und dolmetschen, über Studierende, die Rechtsberatung anbieten, bis hin zu Engagierten, die faktisch sozialpädagogisch tätig werden (z.B. Schäuble 2017; AWO 2015; Petersen 2018). Die stellvertretende Landrätin im brandenburgischen Landkreis B betont: Die Flüchtlinge „müssen Sprache lernen können, die Kinder müssen in die Kita, die müssen in die Gesellschaft sozusagen auch integriert werden, sie brauchen Beratung, sie brauchen Begleitung [...]. Aber sagen Sie mir die Institutionen, die diese Beratungsangebote jetzt ausreichend finanzieren? Gibt es nicht. Also sprich, da setzt man darauf, dass die Gesellschaft sich da selber organisiert, dass man sozusagen im Sinne von Nachbarschaftshilfe jetzt da eben Unterstützung gibt. Und ich denke, das ist aus meiner Sicht eben irgendwo eine Form der Ausnutzung.“ (Jutta Keit, Z. 186ff.) Auch Engagierte benennen Praktiken der Indienstnahme und Prozesse der De-Professionalisierung, wobei dies nicht immer in problematisierender Absicht geschieht und mitunter mit einem gewissen Stolz verbunden ist: „Ich mache mittlerweile nicht mehr Flüchtlingshilfe, sondern ich mache Sozialarbeit", betont eine Engagierte im selben Landkreis (Helene Kolb, Z. 444f.), wobei sie häufig Aufgaben übernehmen würde, ,die eigentlich die Mitarbeiter der Einrichtung machen müssten“ (Z. 670f.).

Eingebettet ist diese Entwicklung in einen Prozess der „Verzivilgesellschaftlichung der sozialen Frage“ (van Dyk und Haubner 2021), der in der Forschung zu Flüchtlingshilfe nur selten in seiner vollen gesellschaftlichen Tragweite thematisiert wird. Angesichts sozialstaatlicher Umstrukturierungen sowie einer Krise der sozialen Reproduktion (Jürgens 2010) in Zeiten demografischen und familialen Wandels

\footnotetext{
7 Wir zitieren hier und im Folgenden exemplarisch diverse Quellen aus dem Korpus der Dokumentenanalyse, um die die Heterogenität der Quellen abzubilden; die präsentierten Debattenstränge sind das Ergebnis der Auswertung des Korpus anhand des modifizierten Kodierparadigmas der Grounded Theory.
} 
gewinnt das Potenzial nicht entlohnter Sorgearbeit jenseits familiärer Kontexte politisch an Bedeutung. Dabei geht es nicht nur um entstehende Versorgungslücken durch den Rückbau wohlfahrtsstaatlicher Leistungen, sondern auch um neue Bedarfe, zum Beispiel in der Kinderbetreuung oder der (Alten-)Pflege (Haubner 2017) wie auch in der Flüchtlingshilfe und Integration. Obwohl die sozialen Dienstleistungen in diesen Feldern weiterhin einen wachsenden Sektor darstellen und auch einen entsprechenden Fachkräftebedarf zeitigen, entsteht mit den neuen Entwicklungen, Angeboten und Rechtsansprüchen eine Versorgungslücke, da die Finanzierung von deren professioneller Umsetzung nicht gewährleistet ist. In einer Studie zur staatlichen Engagementpolitik rekonstruiert Daniela Neumann eine Entwicklung hin zu „Freiwilligkeit als gesellschaftliche[r] Produktivitätsressource“ (Neumann 2016, S. 137) seit Mitte der 1990er-Jahre. In der Begründung des Gesetzesentwurfs zum Ehrenamtsstärkungsgesetz von 2013 heißt es paradigmatisch: „In Zeiten knapper öffentlicher Kassen gewinnt die Förderung und Stärkung der Zivilgesellschaft an Bedeutung, denn die öffentliche Hand wird sich wegen der unumgänglichen Haushaltskonsolidierung auf ihre unabweisbar notwendigen Aufgaben konzentrieren müssen. Es ist daher notwendig, Anreize für die Bereitschaft zum bürgerschaftlichen Engagement zu stärken.“ (Deutscher Bundestag 2012, S. 8) Zu beobachten ist hier eine Neuinterpretation des Sozialstaatsgebots, die verstärkt auf informelle Hilfe-, Unterstützungs- und Verantwortungssysteme setzt: Die wachsende Bedeutung von Engagement und Freiwilligenarbeit ist Teil einer Umdeutung der sozialen Frage in eine Frage der fürsorglichen Gemeinschaft. Das, was zunächst schön klingt Gemeinschaftlichkeit, neues Miteinander, Kultur des Helfens - basiert allerdings auf der Einschränkung oder der Verweigerung sozialer Rechte und revitalisiert Formen des Helfens, die durch persönliche Abhängigkeit und Ungewissheit für die Hilfsbedürftigen geprägt sind (van Dyk und Haubner 2019). Nicht nur im Zweiten Engagementbericht der Bundesregierung wird das Engagement zudem als kostengünstige Ressource jenseits arbeits- und sozialrechtlicher Absicherung gepriesen: „Engagierte erzielen einen finanziellen Nutzen und Organisationen schaffen sich eine im Vergleich zu sozialversicherungspflichtiger Beschäftigung kostengünstigere Personalressource, die zugleich die Wettbewerbsfähigkeit freigemeinnütziger Träger in bestimmten Märkten erhöht.“ (Deutscher Bundestag 2017, S. 290) Gefragt ist gerade auf kommunaler Ebene eine große Bandbreite von Tätigkeiten, wie eine „Ausschreibung“ der Kölner Freiwilligenagentur im Herbst 2019 zeigt, mit der Ehrenamtliche für folgende Aufgaben gesucht werden: „Betreuung und Begleitung von Heimkindern, Sprachkurse für Migranten, Frühstückszubereitung in einem Backpacker-Hostel, Grünanlagenpflege und Gerätewartung in einem Sportverein, Transport von Mittagessen zwischen verschiedenen Kindertagesstätten, Kontakte und Besuche im Altenzentrum, Krankenhaus-Besuchsdienste“ (Pinl 2010, S. 12).

Auch wenn die ehrenamtliche Flüchtlingshilfe in diese Entwicklung einzuordnen ist und die große Aufmerksamkeit für sie dazu beigetragen hat, Schattenseiten freiwilliger Hilfe in den öffentlichen Diskurs zu holen, zeigen sich doch einige Besonderheiten dieses Engagementfeldes. Vor allem und wie im vorherigen Kapitel gezeigt, wird ein instrumenteller Zugriff auf die zivilgesellschaftliche Unterstützung für Geflüchtete in bestimmten Kontexten durch rassistische, flüchtlings- und integrationsfeindliche Positionen konterkariert. Häufiger als in anderen Bereichen wird die 
Legitimität der Hilfe-Empfänger*innen in Frage gestellt und sehen sich Helfende mit Kritik oder sogar Bedrohung konfrontiert, wodurch selbst karitatives Engagement in der Flüchtlingshilfe oft eine dezidiert politische Note bekommt. Auch die Rolle des Staates stellt sich vielschichtiger dar als z.B. im Bereich des Pflegeengagements oder der ehrenamtlichen Nachmittagsbetreuung in Ganztagsschulen: Es geht hier nicht ausschließlich um den Staat als Wohlfahrtsstaat in seiner Funktion als Verantwortungs- und Gewährleistungsträger der sozialen Infrastruktur und Daseinsvorsorge, sondern auch um den Staat als hoheitlichen Staat, der über Status, Aufenthaltsrecht und Abschiebung entscheidet. Während einerseits seit 2015 am laufenden Band Preise und Ehrungen für Engagierte in der Flüchtlingshilfe vergeben wurden, werden insbesondere Aktivitäten der Flüchtlingsselbstorganisierung, die Seenotrettung sowie Kampagnen zum Aufenthaltsrecht und gegen Abschiebungen politisch nicht willkommen geheißen und häufig sogar kriminalisiert. ${ }^{8}$

Der „lange Sommer der Migration“ (Hess et al. 2017) hat in diesem komplexen Setting eine Entwicklung angestoßen, die - wie unsere Interviews zeigen - als Prozess der Verschränkung multipler Grenzüberschreitungen verstanden werden kann. Bislang nicht untersucht ist, wie sich die Sondersituation des Sommers 2015 und die ihr eigenen Grenzüberschreitungen in die Verstetigung des Engagements für Geflüchtete und längerfristige Perspektiven und Praktiken von Engagierten eingeschrieben haben.

\subsection{Multiple Grenzüberschreitungen der Engagierten}

Während das überlastungsbedingte Überschreiten der Grenzen von Engagierten auch in den analysierten politischen, zivilgesellschaftlichen und medialen Dokumenten eine zentrale Rolle spielt, sind Analysen und Verlautbarungen, die das Verhalten und die Praxis von Engagierten (v.a. gegenüber Geflüchteten und Fachkräften) problematisieren, die Ausnahme. Neben einigen kritischen Beiträgen in der ,tageszeitung“ (taz) ist hier eine vom Bundesinnenministerium in Auftrag gegebene Studie zur Kooperation von Haupt- und Ehrenamtlichen in der Flüchtlingshilfe zu nennen, die in Interviews mit hauptamtlichen Koordinator*innen den Typus der ,problematischen Ehrenamtlichen“ identifiziert, die sich durch „Grenzüberschreitungen aller Art, vor allem Distanzlosigkeit und ,Übergriffigkeit“ gegenüber Geflüchteten, anderen Ehrenamtlichen und Hauptamtlichen“ auszeichnen (BMI 2018, S. 31). In unserer Analyse geht es weniger darum, einen spezifischen Typus herauszuarbeiten, als darum, konkrete Praktiken der Grenzüberschreitung und ihre Begünstigungs- und Bedingungsfaktoren in den Blick zu nehmen. Dies ist umso bedeutsamer, als negative oder ambivalente individuelle und gesellschaftliche Folgen von freiwilligem Engagement auch wissenschaftlich ein Forschungsdesiderat darstellen (Simonson und Vogel 2020, S. 2). Einschlägige Analysen gehen oft mit einer ,idealistische[n] Unterstellung“ (Dahme und Wohlfahrt 2009, S. 245) einher, indem die Gemeinwohl-

\footnotetext{
8 Siehe dazu die Pressemitteilungen von Proasyl von 2019 (https://www.proasyl.de/news/kriminalisierungder-zivilgesellschaft-jetzt-auch-in-deutschland/; zugegriffen: Aug. 2021) und Amnesty International Deutschland 2020 (https://www.amnesty.de/allgemein/pressemitteilung/europa-und-zentralasien-europasolidaritaet-mit-menschen-auf-der-flucht; zugegriffen: Aug. 2021).
} 
orientierung und die positive Wirkung des Engagements ohne Prüfung vorausgesetzt werden. Während es an kritischen Analysen zum aktivierenden Sozialstaat, die auch die Indienstnahme von zivilgesellschaftlichem Engagement adressieren, nicht mangelt (z.B. Lessenich 2008; Rose 2000), sind empirische Untersuchungen ehrenamtlicher Betätigung, die keinem affirmativen Grundverständnis folgen, nach wie vor eine Seltenheit. ${ }^{9}$ Die idealistische Unterstellung zu überwinden, bedeutet umgekehrt natürlich keineswegs, dass zivilgesellschaftliches Engagement nicht viele Vorzüge hat und Freiräume schaffen kann, um Formen der alltäglichen Solidarität zu stärken. Nicht nur wir machen wiederholt die Erfahrung, dass Kritik an Bedingungen und Praktiken ehrenamtlichen Engagements es erfordert, dies stets zu betonen was letztlich den diagnostizierten affirmativen Überschuss in der Forschung nur

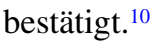

Die im Folgenden dargelegten multiplen Grenzüberschreitungen - die selbstüberschätzende Überschreitung von Professionsgrenzen; politisch motivierte Grenzüberschreitungen in der Interaktion mit Vertreter*innen von Staat und Bürokratie; Grenzüberschreitungen gegenüber Geflüchteten; Überschreiten eigener Grenzen - stellen analytische Verdichtungen im Anschluss an unsere Auswertung der Engagierten- und Expert*inneninterviews dar, die mit exemplarischen Zitaten illustriert werden. Als sensibilisierendes Konzept für die empirische Untersuchung, mit Hilfe dessen gezielt auch Probleme und Fallstricke des Helfens in den Blick kommen, diente die Diagnose des ,voluntary failure“ (Salamon 1987). Diese benennt strukturelle Probleme, die auftreten können, wenn Freiwillige für lebens- und teilhabenotwendige kulturelle und soziale Dienstleistungen verantwortlich sind: Als ,philanthropic insufficiency“, „philantropic particularism“, ,philantropic paternalism“ und ,,philantropic amateurism“ (ebd., S. 39 ff.) werden die Probleme der mangelnden Flächendeckung, der partikularistischen Ausrichtung an subjektiven Prioritäten der Freiwilligen, des Paternalismus (bei Ermangelung sozialer Rechte) sowie der fehlenden Professionalität der Angebote thematisiert.

\subsubsection{Selbstüberschätzung und Verantwortungsanmaßung: Überschreitung von Professionsgrenzen}

Fast alle von uns interviewten Expert*innen berichten, dass sich Engagierte in der Flüchtlingshilfe häufig sowohl über ihre Rolle als engagierte Laien hinwegsetzen als auch Einschätzungen und Ratschläge von professionellen Kräften ignorieren. Diese Selbstüberschätzung kann mitunter zu Fehleinschätzungen und -beratungen führen und im sensiblen Bereich des Aufenthaltsstatus nachteilige Auswirkungen für Geflüchtete haben. So berichtet ein Studierender der Sozialen Arbeit mit Schwerpunkt Engagement: „Also grad wie in so den ausländerrechtlichen Sachen, Anträge

\footnotetext{
9 Eine Ausnahme stellt die Erforschung der sogenannten ehrenamtlichen „Almosenökonomie“ - insbesondere in Gestalt der Tafeln - dar, zu denen mehrere Studien vorliegen, die den Gegenstand im Strukturwandel des Wohlfahrtsstaats verorten (Selke 2009; Kessl und Wagner 2011).

10 Glücklicherweise muss auch nicht (mehr) jede Problematisierung geschlechterhierarchischer Arbeitsteilung hervorheben, dass Sorgearbeit (auch) etwas Schönes sein kann und die meisten Mütter ihre Kinder lieben. Es bleibt zu hoffen, dass dies irgendwann auch für kritische Analysen zu Engagement und Freiwilligenarbeit gelten wird.
} 
ausfüllen oder irgendwas beantragen, das können einfach viele wirklich gar nicht. Und machen es dann halt komplett falsch. Und meinen dann, sie meinen es gut.“ (Gruppendiskussion Studierende, Z. 275 ff.) In anderen Fällen bitten Engagierte bei Behörden unter Vernachlässigung datenschutzrechtlicher Bestimmungen um die Herausgabe personenbezogener Daten Geflüchteter oder mischen sich in Aufgaben professioneller Kräfte ein. So beklagt der Engagementbeauftragte der badenwürttembergischen Stadt D, Engagierte würden immer wieder ,,von sich aus, in Anführungszeichen, Grenzen überschreiten. Und sich in Themen einmischen, weil sie denken, dem Menschen was Gutes tun zu sollen, wo es dann wirklich schwierig wird, wenn die dann anfangen, bei Anwälten anzurufen. Oder bei Gerichten oder bei der Agentur für Arbeit und dann plötzlich vier, fünf Leute an irgendwas beteiligt sind." (Markus Langbach, Z. 786ff.) Niemand bezweifelt den guten Willen der Engagierten, zugleich mache dieser es schwierig, das häufig zu beobachtende „Überengagement“ zu bremsen, das - so etwa die Koordinatorin der Flüchtlingshilfe der Diakonie im brandenburgischen A - aus einer Überidentifikation mit der Rolle als Helfer*in und einer Überschätzung der eigenen Kompetenzen resultiere. „Nur gut gemeint reicht nicht aus“, heißt es entsprechend in der „taz“, wo Hauptamtliche - wie die Referentin für freiwilliges Engagement beim Diakonischen Werk Hamburg - auf die Problematik von „Hilfe ohne geschulte Unterstützung“ (Diemand 2015) hinweisen. Im Sinne des sensibilisierenden Konzepts des „,voluntary failure“ (Salamon 1987, S. 39) erweist sich hier die Problematisierung eines ,philantropic amateurism“ als zentraler Kritikstrang.

Ein zweiter Blick vermag allerdings zu zeigen, dass der wesentliche Grund für die konstatierten Kompetenzüberschreitungen oft nicht in der persönlichen Hybris der Engagierten, sondern in den Rahmenbedingungen der Nothilfe 2015 und 2016 liegt. In Ermangelung von professionellen und hauptamtlichen Kräften haben Freiwillige behelfsmäßig deren Aufgaben übernommen bzw. übernehmen müssen - und sich notdürftig selbst geschult und in der Praxis angelernt. Dies gilt, wie unsere Interviews zeigen, auch für Engagierte, die eigentlich der festen Überzeugung sind, dass ihre Aufgaben in den Zuständigkeitsbereich des Sozialstaates fallen und gerade nicht freiwilligen Laien überlassen sein sollten. Das Staatsversagen in Reaktion auf den ,langen Sommer der Migration“ spielt insofern eine strukturell entscheidende Rolle dafür, dass Engagierte auch über diese unmittelbare Ausnahmesituation hinaus etablierte Engagementgrenzen überschreiten - und zwar in eingeübter Praxis auch dort, wo Hauptamtliche übernehmen (sollen).

Der Leiter einer Fortbildungsakademie für Engagierte im Bundesland Brandenburg unterstreicht, dass es sich als recht konfliktreich erweist, in der Verstetigung der Flüchtlingshilfe die Grenzen zwischen Engagement und professioneller Expertise zu (re-)etablieren: „Es gibt Personen, die nun glauben, nachdem sie sozusagen drei Jahre Geflüchtete begleitet haben, dass sie viele Dinge wesentlich besser wissen als Fachkräfte. Und auch wenn ich glaube, dass es da eine Entwicklung von Erfahrungen und auch von Kompetenzen gegeben hat, aber es ersetzt nicht die Fachkräfte.“ (Werner Kehrer, Z. 175 ff.) Die Koordinatorin der Flüchtlingshilfe der Diakonie im brandenburgischen A betont dazu: „Also es gab wirklich anderthalb Jahre, wo jeder Professionelle sagen würde, ohne die Ehrenamtlichen hätten wir es nicht geschafft. Keine Frage, ja. Aber jetzt kommen wir in eine Phase [...], dass die 
Ehrenamtlichen eigentlich wieder was abgeben müssten. Dass es halt auch keine Fehlberatung gibt und dass die professionellen Strukturen eigentlich auch besser greifen.“ (Monika Stemmert, Z. 417 ff.) Derlei Konflikte dürften zusätzlich durch den Umstand verstärkt werden, dass viele der innerhalb recht kurzer Zeit neu eingestellten Hauptamtlichen ihrerseits gerade erst das Studium beendet hatten und angesichts des großen Bedarfs oft (noch) nicht einschlägig für die konkreten Tätigkeitsfelder qualifiziert waren, während Engagierte oftmals über mehrjähriges informelles Erfahrungswissen verfügen (Mutz und Wolff 2018, S. 61). Erschwert wird eine konsequente Grenzziehung aber auch dadurch, dass Verwaltungen Geflüchtete mitunter selbstverständlich mit rechtlichen Fragen oder medizinischen Anliegen an ehrenamtliche Strukturen weitervermitteln, wie Engagierte aus dem Unterstützerkreis Asyl im baden-württembergischen C, den Refugee Law Clinics und den Büros für medizinische Flüchtlingshilfe berichten. Eine Arbeitsvermittlerin des Jobcenters im Landkreis B, die Geflüchtete in enger Kooperation mit Engagierten in den ersten Arbeitsmarkt vermittelt, bemerkt hierzu: „Und da sind die Ehrenamtler eben für mich die Mittler und auch die Helfer und die Zwischenstellen dazwischen. Und damit entlasten sie uns.“ (Anja Sehlen, Z. $560 \mathrm{ff.)}$

Zusätzlich scheint die breit berichtete Kompetenzüberschreitung durch einen für diesen Engagementbereich typischen Mittelschichts-Bias der Engagierten, die überdurchschnittlich qualifiziert und gebildet sind, befördert zu werden (Mutz und Wolff 2018, S. 56; BMFSFJ 2017, S. 22). So finden sich hier Akademiker*innen, die sich berufsnah engagieren und die damit tatsächlich über einschlägige Kompetenzen verfügen. Das wiederholt berichtete Beispiel sind pensionierte Deutschlehrer*innen in ehrenamtlichen Deutschkursen, die sich auch in unserem Sample finden und die teilweise über Anzeigen in Lehrerzeitungen gewonnen wurden (Memarnia 2015). Aber auch im medizinischen und psychotherapeutischen Bereich haben sich viele einschlägig Qualifizierte insbesondere in der Hochphase der Nothilfe ehrenamtlich engagiert (Müller 2016). In diesen Fällen ist weniger eine De-Professionalisierung des Engagements als eine Informalisierung von Bildungs- und Betreuungsangeboten zu konstatieren, da fachlich qualifizierte Engagierte eine professionelle Leistung unter nicht regulierten, nicht erwerbsförmigen Bedingungen anbieten, was zu ganz neuen Problemen im Verhältnis von Haupt- und Ehrenamt führen kann. Zudem berichten einige Interviewte bei nicht berufsnah engagierten Akademiker*innen von AnmaBungen, die weniger fachlich begründet sind, als dass sie mit dem Selbstbewusstsein von Akademiker*innen einhergehen: So sei eine Resistenz gegen Schulungs- und Weiterbildungsangebote zu beobachten, die mit einem bestimmten Gestus - ,ich weiß doch, wie die Dinge zu händeln sind“ (Werner Kehrer, Z. 160) - zurückgewiesen werden. Ein Interviewter berichtet zudem von Engagierten, die ,zum Teil Juristen, Ärzte oder sonst was [sind], und wenn sie da mit denen praktisch: ,Das ist mein Sohn aus Gambia... 'Gehen da zu der Ausländerbehörde und sind da auch laut und keine Ahnung. Fühlen die sich alle irgendwie bedrängt.“ (Gruppendiskussion Studierende, Z. 298 ff.) Hier sticht neben dem Status der Engagierten eine ,Anwaltschaft" für die Geflüchteten ins Auge, die gleichermaßen - wie im Folgenden zu zeigen sein wird - rebellische wie paternalistische Züge tragen kann.

Wiederholt kommunizieren Hauptamtliche in den Interviews die Schwierigkeit, Engagierte zu kritisieren oder Aspekte des Engagements zu problematisieren. Hier 
zeigt sich ein Problem, dass in der Auseinandersetzung mit den Schattenseiten ehrenamtlicher Betätigung insgesamt zu beobachten ist: eine Sakralisierung des Engagements mit selbstlosen Engagierten als Protagonist*innen (Eliasoph 2013, S. $2 \mathrm{f}$.), die eine kritische Reflexion ehrenamtlicher Arbeit erschwert. Darauf deuten auch die diesbezüglichen Leerstellen unserer Dokumentenanalysen hin: Paternalismus gegenüber Geflüchteten oder Problematiken fachlich nicht qualifizierter Hilfe finden hier so gut wie keine Erwähnung. Die Debatte über Engagement und Freiwilligenarbeit ist in politischen, wohlfahrtsverbandlichen, kirchlichen und medialen Beiträgen mehrheitlich geprägt von einem kollektiven Lob der Engagierten, die wiederholt als „Alltagshelden“ (Deutscher Bundestag 2017, S. 393) und „Engel“ (Der Paritätische 2014, S. 33) adressiert werden. „Das Ehrenamt allgemein wird schon als heilig angesehen hier in Deutschland" betont auch eine Studierende der Sozialen Arbeit in einer Gruppendiskussion (Z. 315 f.). Während einige der von uns interviewten Expert*innen diese Konstellation kritisch sehen, bleiben zwei wesentliche Aspekte allerdings oft unterbelichtet: erstens, dass die staatliche Politik der Indienstnahme freiwilliger Hilfe für lebensnotwendige und teilhabesichernde Infrastruktur eben diese Grenzüberschreitung angereizt hat und ausbeutet; und zweitens, dass beklagte „Anmaßungen“ von Engagierten nicht selten auf die nachvollziehbare Kritik am staatlichen Umgang mit Geflüchteten zurückzuführen sind.

\subsubsection{Eigensinn des Engagements: Politisch motivierte Grenzüberschreitungen gegenüber Hauptamtlichen}

Die Übergänge zwischen fachlichen Grenzüberschreitungen durch nicht einschlägig qualifizierte Laien einerseits und Grenzüberschreitungen im Sinne einer eigensinnigen, politischen Anwaltschaft für Geflüchtete andererseits können fließend sein. Hier kommt der dem zivilgesellschaftlichen Engagement stets zugeschriebene Eigensinn zum Tragen (z.B. Klein 2016), der diesem eine besondere Eigenlogik und Handlungsspielräume zuschreibt, die hauptamtliche Arbeit - zumal im Behördenund Verwaltungskontext - nicht haben kann. Die in der Flüchtlingshilfe aktive Pfarrerin Julia Rau im brandenburgischen Landkreis B weist zum Beispiel darauf hin, dass Engagierte in besonderer Weise Vertrauenspersonen von Geflüchteten sein können, da sie anders als viele Hauptamtliche nicht jeden Regelverstoß zur Anzeige bringen müssen. Hier wird die Grenzüberschreitung als politische Anwaltschaft verteidigt und als Stärke der ehrenamtlichen Unterstützung hervorgehoben. Serhat Karakayali (2017, S. 22) betont auf Basis eigener empirischer Erhebungen: „Ehrenamtliche agieren als ,Anwälte“ der Geflüchteten und erkämpfen Leistungen, die BehördenmitarbeiterInnen verweigern oder die Geflüchteten gar nicht erst angeboten werden.“ In der „taz“ wird zudem das rebellische Potential des Engagements hervorgehoben, dessen Stärke gerade darin liege, sich institutionellen Schranken widersetzen zu können: „Anders als in der Verwaltung gibt es für Solidarität keine Richtlinien und keine Kontrolle.“ (Jakob 2015)

An diesem Punkt offenbart unser Material ein Spannungsverhältnis von Hauptund Ehrenamt, denn nicht wenige Hauptamtliche beklagen, dass Engagierte mit dem Verweis auf den ,gesunden Menschenverstand“ gegen fachliche Vorgaben und Verfahrensstandards verstoßen würden. So betont die Leiterin eines Integrations- und 
Beratungszentrums in Baden-Württemberg: „Die Ehrenamtlichen, die sich dort in der Flüchtlingshilfe engagiert haben, kannten das ganze Themengebiet nicht. [...] Die kannten die gesetzlichen Regelungen nicht. Und sind mit dem gesunden Menschenverstand rangegangen. [...] Und haben nicht verstanden, warum das Hauptamt anders reagiert.“ (Birgit Kress, Z. $691 \mathrm{ff}$.) Dies wirft die wichtige Frage auf, wann der „gesunde Menschenverstand“ zu fachlich unpassendem, fehlerhaftem Handeln führt, das die Situation von Geflüchteten unter Umständen gefährdet, und wann er umgekehrt das Potenzial hat, problematisches, mitunter auch rassistisch konnotiertes (haupt-)amtliches Handeln eigensinnig herauszufordern - eine Frage, die wohl nur im Einzelfall zu entscheiden ist. Wann wird der Eigensinn zur unqualifizierten Anmaßung? Und wann delegitimiert die vordergründig fachlich motivierte Kritik von Hauptamtlichen das kritische Potenzial des Engagements, das eben auch dazu beitragen kann, behördlichen Rassismus, ,Schlampigkeit der Behörden“ (Rolf Thoma, Z. 266) und die Nichtnutzung von Ermessensspielräumen öffentlich zu machen?

Tatsächlich argumentieren die von uns interviewten Engagierten in vielen Fällen nicht einfach mit dem ,gesunden Menschenverstand“, sondern auf Grundlage einer differenzierten Kritik des Umgangs mit Geflüchteten und Asylsuchenden in Deutschland, den sie in ihrem Engagement hautnah erleben; ihr Engagement begreifen sie vor diesem Hintergrund als ,eine Art Notwehr“ (Ludwig Santner, Z. 751) gegen die mangelhafte staatliche Integrationspolitik. In diesem Sinne zeigt auch die Analyse der Orientierungsrahmen der Engagierten, dass sich in der Flüchtlingshilfe viele finden, die sich - anders als in anderen Engagementbereichen - gar nicht vorrangig als Ehrenamtliche, sondern als politische Aktivist*innen sehen. Aus dieser Perspektive sind gerade nicht Professionalität und Regelkonformität, sondern die Anwaltschaft und Solidarität unter Bedingungen staatlicher Unterversorgung, Ausgrenzung und Repression entscheidend; ,, besser unprofessionelle Unterstützung als professionelle Ausgrenzung und Abschiebung“, ließe sich diese Position zuspitzen.

\subsubsection{Viktimisierung und Paternalismus: Grenzüberschreitungen gegenüber Geflüchteten}

Die konsequente Anwaltschaft kann jedoch auch mit einer paternalistischen Haltung gegenüber Geflüchteten einhergehen. Schon lange weisen Geflüchtete darauf hin, dass die Kultur des Helfens oftmals koloniale Muster der Überlegenheit reproduziert, so zum Beispiel der Aktivist Bino Byansi Byakuleka: „Die meisten sind zufrieden damit zu ,helfen“. Sie wollen uns nicht als menschliche Wesen sehen, die die gleichen Rechte haben. Sie wollen ihre europäische Helferidentität aufrechterhalten und uns dadurch abwerten." (Byakuleka 2016, S. 18) ${ }^{11}$ In Anbetracht eines ungesicherten Aufenthaltes, der abgestuften sozialen Rechte, oftmals traumatischer Fluchterfahrungen sowie eines verbreiteten Alltagsrassismus ist der gesellschaftspolitische Horizont, in dem die Flüchtlingshilfe agiert, in besonders ausgeprägter Weise durch Hierarchien geprägt, die sich auch zwischen Helfenden und Geflüchteten ma-

11 Für eine dekoloniale Perspektive vgl. auch Braun (2017, S. 42f.): „A decolonial approach renders visible the way in which these historical and colonial sedimentations surface in contemporary welcome culture." 
nifestieren (Jungk 2016; van Dyk 2019). ${ }^{12}$ Auch die von uns Interviewten benennen diese Problematik - oder argumentieren umgekehrt in einer Weise, die Geflüchtete zu passiven Empfänger*innen von Hilfe und Anleitung macht. In einer Gruppendiskussion mit Alltagsbegleiter*innen in der Flüchtlingshilfe in Brandenburg betont ein Teilnehmer die grundsätzliche Überlegenheit der deutschen Helfer*innen: „Sie kriegen ja die Information oder Rat von Deutschen letzten Endes, also von Leuten, die hier wohnen, die sich hier auskennen, die ja wissen, was sie sagen. Wir sind ja keine Blöden sozusagen, also wir haben ja Gründe. Wenn ihnen der Syrer einen Rat gibt, der kann ja alles Mögliche raten.“ (Z. $717 \mathrm{ff.)}$

Eine Engagierte im brandenburgischen Landkreis B bemängelt gerade in kirchlichen Kontexten eine paternalistische Form der Hilfe, stelle sie doch ,bei den kirchlichen Helfern häufig fest, dass da diese Einstellung [ist]: ,Ach Gott, das sind arme Menschen, die müssen wir unterstützen“" (Helene Kolb, Z. 599ff.) Teilnehmer*innen einer Fortbildung zum Beziehungsengagement (z.B. Patenschaften oder Sprachtandems) problematisieren, dass viele Engagierte dazu neigten, die Geflüchteten zu infantilisieren, zu bevormunden und ihnen ihre Individualität abzusprechen. In der Diskussion wird eine große Sensibilität für Hilfe als ,relation of inequality“ (Fassin 2012, S. 3) erkennbar. Die Leiterin einer Fortbildung der Ehrenamtsakademie Berlin unterstreicht ebenfalls: „Aber es gibt es halt immer wieder, dass man so sprachlich so ,mein Geflüchteter' [...], und da ist es viel passiert, dieses ,ich weiß, was für dich gut ist". (Ursula Vollmer, Z. $120 \mathrm{f}$.) Auch die Leiterin eines Workshops zu Selbstfürsorge im Ehrenamt und eine Supervisorin mit dem Schwerpunkt „Burnout und Ehrenamt“ benennen Paternalismus und Viktimisierung von Geflüchteten als zentrale Probleme, werde die Hilfe doch für viele Engagierte mitunter zum Selbstzweck und zur Quelle der Bestätigung der eigenen Wichtigkeit. Nicht selten würden dabei die Wünsche der Geflüchteten übergangen und eigene (normative) Vorstellungen etwa in Bezug auf kulturelle Integration durchgesetzt. Neben dem Paternalismus zeigt sich hier auch eine Tendenz zum ,philantropic particularism“ (Salamon 1987, S. 39), der einseitig an den Interessen der Engagierten ausgerichtet ist.

Auch in unserem Sample gibt es zahlreiche Hinweise darauf, dass „Helferinnen und Helfer davon überzeugt [sind], dass das eigene Entwicklungsmodell das bestmögliche ist. All das spricht nicht gegen Hilfe, aber umso mehr für eine reflexive Hilfe, die um ihre Grenzen weiß." (Gebauer 2016) Wie herausfordernd eine solche Reflexion und Gratwanderung ist, sehen wir in unserem Sample an zwei weiblichen Flüchtlingshelferinnen, die versuchen, in die Unterstützung ihre Vorstellungen von Emanzipation einfließen zu lassen, obwohl diese nicht notwendigerweise mit den Vorstellungen der Geflüchteten übereinstimmen. Eine Seniorenbeirätin im brandenburgischen Landkreis B möchte beispielsweise einer geflüchteten Mutter im Rahmen ihrer eigenen, durch ihre DDR-Sozialisation geprägten Vorstellungen von Familien-

\footnotetext{
12 Reproduziert wird diese Hierarchie auch in der medialen Berichterstattung über die Flüchtlingshilfe, in der Geflüchtete selten zu Wort kommen, während die Perspektiven von Engagierten großen Raum einnehmen. Auch bei den zahlreichen politischen Würdigungen des Engagements tauchen die Geflüchteten so gut wie nie als aktive Subjekte auf, die sich selbstorganisiert für ihre Rechte einsetzen, obwohl es zahlreiche entsprechende Beispiele gibt (z.B. Steinhilper 2017).
} 
planung helfen: ,Jetzt ist Schluss mit Kinder machen. So, sage ich, jetzt gehen wir mal wieder zum Frauenarzt. Ich war zum Frauenarzt mit ihr. [...] Eine Totaloperation wird nicht bezahlt, aber wie nennt man das, was man sich einsetzt? Spirale und dann hier so einen Stuck. [...] ,Haben wir auch alle. Das machen wir auch.' [...] Die hat ihre Spirale drin, die bekommt keine Kinder mehr.“ (Berta Gelbcke, Z. $241 \mathrm{ff}$.)

Ein Mehr an Reflexion allein kann allerdings kaum aus der hierarchischen Konstellation und dem der Hilfebeziehung zugrundeliegenden Abhängigkeitsverhältnis herausführen. Die freiwillige Hilfe kann - das ist ihr Wesensmerkmal - ,jederzeit ohne Angabe von Gründen verweigert oder eingestellt werden, sie ist hochgradig abhängig von Stimmungen, Emotionen und Sympathien“ (van Dyk 2019, S. 39). Während wohlfahrtsstaatliche Versorgungslücken im Blick auf die Instrumentalisierung, Überforderung und Überlastung Engagierter breit diskutiert worden sind, bleibt die Position und Perspektive der Geflüchteten als Adressat*innen der Hilfe in der öffentlichen Debatte unterbelichtet. Sehr treffend werden die Hierarchien und Fallen des Helfens in einem Kommentar der „taz“ - eine der raren Verlautbarungen in dieser Frage - auf den Punkt gebracht: „Nur zwei Dinge schützen gegen diese Fallen: Reflexion aufseiten der Helfer und Autonomie aufseiten der Flüchtlinge. Als Faustregel könnte gelten: Je stärker die Grundbedürfnisse gesichert sind, desto weniger Probleme gibt es. Wenn Flüchtlinge ausreichende Sozialleistungen bekommen, ein Dach über dem Kopf haben und vor Abschiebung geschützt sind, können sie leidlich frei entscheiden, ob sie sich zum Amt begleiten oder für ein Theaterstück casten lassen, zum Deutschkurs der Heim-Anwohner gehen oder das aufgemöbelte Kinderfahrrad als Geschenk annehmen wollen.“ (Jakob 2015) Dass genau die genannten Bedingungen gesicherter Grundbedürfnisse gesellschaftlich hoch umstritten sind, zeigen nicht zuletzt auch unsere Interviews mit Engagierten aus anderen Bereichen, von denen Geflüchtete gerade nicht als gleichberechtigte, legitime Adressat*innen von Rechten, Leistungen und Hilfen angesehen werden.

\subsection{4 Überlastung und Überforderung: Vom Überschreiten eigener Grenzen}

Es lässt einen nicht mehr los, abends, wenn man schlafen gehen will oder so. [...] Und dann merke ich dann selber, jetzt wird es mit mir kritisch. Jetzt muss ich aufpassen. Das geht dann wahrscheinlich auch irgendwann an die Gesundheit. (Rolf Thoma, Engagierter im baden-württembergischen C, Z. 383 f.)

Über die Überlastung der Engagierten in der Flüchtlingshilfe und den schwierigen Umgang mit der zum Teil großen Verantwortung ist medial wie wissenschaftlich viel geschrieben worden (z.B. Mutz et al. 2015; Deggerich et al. 2015; Lokalzeitung Stadt C 2017). Alle der von uns interviewten Haupt- und Ehrenamtlichen sprechen davon, dass viele Engagierte nach den ersten Monaten und der allseits konstatierten „regelrechten Euphoriewelle im Spätsommer 2015“ (Beckmann et al. 2018, S. 38) ausgebrannt und erschöpft gewesen seien und sich zurückgezogen hätten. Die besondere Belastung in der Flüchtlingshilfe wird auch von Interviewten, die in anderen Feldern ehrenamtlich engagiert sind, hervorgehoben: „Die Ehrenamtlichen, die die [Geflüchteten] aufgenommen haben, die die betreut haben. Wo war der Staat? [...] Und haben dann weitergearbeitet, die waren fast bis zur Erschöpfung." (Ute Zeller, 
Ökumenische Sozialstation im baden-württembergischen C, Z. $596 \mathrm{ff}$.) Im badenwürttembergischen Landkreis $\mathrm{C}$ sind nach Angaben des lokalen Integrations- und Beratungszentrums 2017 nur noch 10\% der im Herbst 2015 Engagierten weiterhin aktiv. Die Leiterin des Zentrums betont: „Es gab einfach Situationen, wo das Ehrenamt vielleicht wirklich auch überfordert war. Und einige sind uns dann trotz dieser Unterstützung, die wir gegeben haben, weggebrochen." (Birgit Kress, Z. 342 ff.)

Im Gespräch mit Expert*innen wird deutlich, dass die Überlastung nicht nur den äußeren Rahmenbedingungen und dem großen Bedarf an Unterstützung geschuldet ist, sondern dass vielen Engagierten mit Blick auf psychische Belastungen zudem Erfahrung und Expertise im Umgang mit Grenzziehungen fehlen. Eine Fortbildung zu Nähe und Distanz im Beziehungsengagement in Berlin, an der wir beobachtend teilgenommen haben, war dementsprechend explizit auf die Aneignung therapeutischer Selbsttechnologien ausgerichtet, um so einen pragmatischen Umgang mit den eigenen Gefühlen im Beziehungsengagement zu fördern. Dabei wurde deutlich, dass es vor allem darum geht, einen plötzlichen Abbruch des Engagements aus Überlastungsgründen zu vermeiden. Zugleich berichten der Leiter einer Fortbildungsakademie für Engagierte sowie die bereits erwähnte Supervisionstrainerin mit Schwerpunkt „Burnout und Ehrenamt“, dass die Nachfrage nach Angeboten der Selbstfürsorge insgesamt - und verglichen mit anderen Schulungsangeboten, z.B. zum Asylrecht - eher gering sei. Beide machen dafür einerseits die Zeitknappheit überlasteter Engagierter verantwortlich, betonen andererseits aber auch den Umstand, dass die Menschen ihr freiwilliges Engagement einfach niederlegen können, wenn es zu belastend wird: „Ich glaube, das liegt daran, dass die Leute denken: [...] Selbstfürsorge brauche ich nicht, weil wenn es mir nicht mehr gefällt, höre ich auf.“ (Irene Schmied, Z. 217f.)

\section{Exit, Voice und Loyalty: Zum Umgang mit Herausforderungen in der Flüchtlingshilfe}

Engagierte in der Flüchtlingshilfe sind mit vielfältigen Herausforderungen konfrontiert - von der Instrumentalisierung ihrer Arbeit über das Ringen mit Behörden und die alltägliche Überlastung bis hin zu Anfeindungen im sozialen Umfeld. Aber auch ihr eigenes Handeln weist mitunter problematische Facetten auf - z.B. in Gestalt paternalistischer oder unprofessioneller Hilfe -, deren Thematisierung durch die verbreitete Sakralisierung von Engagement sowie die Ausblendung staatlicher Indienstnahmepolitiken erschwert wird. Erst die Einordnung in die übergreifende Entwicklung der Verzivilgesellschaftlichung der sozialen Frage, die karitative gegenüber politischen Komponenten der Zivilgesellschaft stärkt, erlaubt es schließlich, die multiplen Grenzüberschreitungen in ihrem Zusammenspiel und ihrer gesellschaftlichen Tragweite zu erfassen. Paternalismus, fehlende fachliche Expertise, Überlastung oder Angebotslücken sind gerade nicht auf das individuelle Versagen einzelner Engagierter zurückzuführen, sondern auf strukturelle Ursachen und die staatlich beförderte Informalisierung von Verantwortung für lebensnotwendige und teilhabesichernde Güter und Infrastrukturen. 
Im letzten Schritt widmen wir uns nun den Umgangsweisen der Engagierten mit den genannten Problemen, für deren Systematisierung wir auf die Modi Exit, Voice und Loyalty zurückgreifen. Diese wurden als übergreifende Verdichtungen aus der vergleichenden Auswertung von Engagierten- und Expert*inneninterviews gewonnen, mit thematisch einschlägigen Codes der Dokumentenanalyse verglichen und mit der einschlägigen Sekundärliteratur angereichert. Die Bezeichnung der drei Modi erfolgt in Analogie zu den Analysen Albert O. Hirschmans (1970), in denen dieser mögliche Reaktionen auf Probleme und Leistungsabfall in sozialen Beziehungen (z.B. Unternehmen, Organisationen) systematisiert hat. Für Hirschman markiert Exit die Abwanderung bzw. die Beendigung einer Zugehörigkeit und Voice die vorgetragene Beschwerde bzw. Kritik, die eine Veränderung der Situation zum Ziel hat, die einen Exit überflüssig macht. Er geht davon aus, dass es von der Loyalität gegenüber der sozialen Beziehung/Organisation abhängt, ob Individuen sich für Exit oder Voice entscheiden.

Wir wenden diese Systematisierung auf den vielschichtigen Kontext der Flüchtlingshilfe an, die natürlich nicht mit einem einzelnen Unternehmen oder einer Organisation zu vergleichen ist; gleichwohl erweist sich das Instrumentarium als hilfreich, um Umgangsweisen mit Herausforderungen und Problemen in der Flüchtlingshilfe zu sortieren und in ihrem Wechselspiel zu beleuchten. Übertragen auf unseren Gegenstand zeigt sich im Sample deutlich, wie eng die Option des Exits mit dem freiwilligen Charakter des Engagements verbunden ist: „Ich habe jederzeit das Gefühl, ich kann sagen: Ade“ (Renate Bucher, Z. 575f.) sagt eine Engagierte. Ein anderer Befragter erwidert auf die Frage, ob das Engagement ihn stresse: „Nicht wirklich, nein. Ich muss ja nichts machen im Rahmen von Engagement, also ich bin ja frei. Kann mich umdrehen und gehen." (Ludwig Santner, Z. 446f.)

Gerade weil der Austritt so niedrigschwellig ist, scheint diese Option indes sowohl eine gute Selbstfürsorge als auch eine Politisierung des Engagements im Sinne einer Problematisierung der Engagementbedingungen zu erschweren. Obwohl dieser Befund für freiwilliges Engagement in seiner Breite gelten dürfte, erweist sich diese Deutungsfolie für die Analyse der Flüchtlingshilfe als besonders geeignet: Zum einen haben wir es hier häufig mit einem kaum institutionalisierten Ad-hoc-Engagement zu tun, das keine langjährige, Loyalität stiftende institutionelle Anbindung aufweist - was den Ausstieg erleichtert. Zum anderen kommt der große Unterschied zwischen der ersten Phase der Nothilfe und der seit Ende 2016 erfolgten Verstetigung des Engagements hinzu. Mit dieser Verstetigung haben sich die Aufgabenfelder, die Komplexität der Aufgaben, das Verhältnis von Haupt- und Ehrenamt sowie die existenzielle Dringlichkeit der Aufgaben stark gewandelt (Beckmann et al. 2018, S. 28) - wodurch in der zunehmenden „Ruhe nach dem Sturm“ (Mutz und Wolff 2018, S. 60) die Hürden für einen Exit zusätzlich gesunken sein dürften. Dass sich im Laufe des ersten Jahres ,die Aufgaben dramatisch geändert [haben]“ (Irene Schmied, Supervisionstrainerin für Ehrenamtliche, Z. $182 \mathrm{ff}$.) - von der Erstversorgung hin zur Unterstützung bei Wohnungssuche und (Arbeitsmarkt-)Integration -, habe zu neuen und oft unklaren Rollen und Rollenverteilungen geführt.

Die niedrigschwellige Option des Exits als möglicher Reaktion auf Anregungen und Kritik erschwert nicht zuletzt auch die Zusammenarbeit zwischen Haupt- und Ehrenamtlichen, wie die Koordinatorin der Flüchtlingshilfe der Diakonie im bran- 
denburgischen A anmerkt: „Wenn ich ganz vorsichtig versuche, mich zu äußern oder mal kritisch zu hinterfragen und so, dann sofort Rückzug." (Monika Stemmert, Z. 352 ff.) Hinzu kommt, gerade bei längerfristig Engagierten, die Erfahrung der (drohenden) Abschiebung der Geflüchteten. Diese wird nicht nur als große Bedrohung für die Geflüchteten selbst erlebt, sondern auch als grundlegende Missachtung des eigenen Engagements und als fundamentale Enttäuschung über den Staat bzw. die ausführenden Organe. Die Sprecherin eines Aktionsbündnisses gegen Rassismus und Diskriminierung in Brandenburg unterstreicht in Übereinstimmung mit anderen Expert*innen im Sample, dass häufig eher mit Rückzug als mit politischer Gegenwehr reagiert werde: „Da haben wir aber auch wahnsinnig viele Anrufe gekriegt, [...] ,ich helfe, ich engagiere mich, und dann wird der abgeschoben'. [...] Und das ist auf alle Fälle ein Thema, wo man sich eher zurückzieht aus dem Ehrenamt, nicht unbedingt politischer deswegen wird." (Hanna Zange, Z. $483 \mathrm{ff}$.)

Doch nicht immer ist Rückzug bzw. Exit der gewählte Weg: Während die institutionelle Loyalität aus den genannten Gründen oft wenig ausgeprägt ist, zeigt sich in unserem Sample bei einigen Engagierten eine starke Loyalität gegenüber den Unterstützungsbedürftigen. Diese wurzelt vor allem in einem Gefühl persönlicher Verantwortung im Beziehungsengagement, wie eine ehrenamtliche Lesepatin bemerkt: „Ja, ich hätte auch ein schlechtes Gewissen, wenn ich jetzt sagen würde: ,Heute komme ich nicht, morgen komme ich, vielleicht übermorgen auch noch, aber dann wieder nicht"; entweder ich sage es zu oder ich sage nicht zu. Und das ist schon wieder die Gewissenhaftigkeit, die man dann hat und die Verantwortung für den anderen." (Karina Lorenz, Z. $421 \mathrm{ff}$.) Gerade im ländlichen Brandenburg führt diese Loyalität zu dem beschriebenen Phänomen der Verheimlichung des Engagements im Fall von Anfeindungen, das sich zugleich einer klaren Zuordnung zu den Modi Exit und Voice entzieht: Hier ermöglicht die Heimlichkeit eine Fortsetzung des Engagements (verhindert also den Exit), setzt der Artikulation von Kritik (Voice) zugleich aber enge Grenzen, insofern diese nicht öffentlich sichtbar werden darf. Es greift also einerseits eine starke Loyalität und Solidarität mit den Geflüchteten, andererseits wird der Preis, öffentlich für diese Loyalität einzutreten, angesichts der erlebten Anfeindungen als zu hoch erachtet.

Parallel finden wir in unserem Sample aber auch Beispiele für Voice im Sinne formulierter Kritik, wobei sich verschiedene Modi von Voice zeigen, die auf unterschiedlichen Voraussetzungen beruhen. Die am wenigsten weitreichende Form des Sprechens und Kritisierens ist (1) der „Druckablass“ in Supervisions- und Schulungsformaten, der - wie unsere teilnehmenden Beobachtungen zeigen - häufig ohne konkrete Veränderungsabsicht bleibt, aber als Ventil für Unzufriedenheit fungiert. Eine solche Umgangsweise finden wir dort, wo eine starke institutionelle Einbindung oder eine ausgeprägt karitative Orientierung vorhanden sind. Weitergehender sind Formen von Voice, die (2) auf Veränderungen der Engagementbedingungen vor Ort zielen, zum Beispiel durch Kritik an fehlenden finanziellen Mitteln oder mangelnder Kooperation der Verwaltung. Auch hier wird die Rolle des Engagements nicht grundsätzlich problematisiert, sondern es werden engagementfreundlichere Bedingungen durch eine bessere materielle Ausstattung, Coaching-Angebote, hauptamtliche Koordinierungsstellen oder (im)materielle Formen der Anerkennung eingefordert, sogenannte „Hilfen für Helfer“ (Stadler 2016). Engagierte Einzelper- 
sonen spielen hier ebenso wie Wohlfahrtsverbände und etablierte Freiwilligenorganisationen eine zentrale Rolle. Eine weitere Form von Voice findet sich (3) in dem dezidiert antirassistischen Verständnis von Engagement, das wir beschrieben haben: Diese Form der Positionierung wird durch Bedingungen befördert, die die Flüchtlingshilfe unter Druck setzen, und häufig von Engagierten verfolgt, die ihre Rolle stärker politisch als karitativ verstehen. Gleichwohl zeigen unsere Interviews, dass die geschilderten schwierigen Bedingungen auch zur Politisierung karitativen Engagements beigetragen haben. Eine engagierte Schülerin im brandenburgischen Landkreis B beschreibt beispielsweise ihre Politisierung mit den Worten: „Ja, also durch die Geflüchtetenhilfe auf jeden Fall halt, weil man sich halt auch so politisch dann halt damit beschäftigt hat und so. Hatte ich halt vorher quasi keine so richtige Meinung dazu.“" (Lena Jung, Z. 498 ff.)

Während Politisierung in diesem Modus die offensive Verteidigung des lokalen Engagements gegen rassistische Anfeindungen und politische Blockaden meint, stehen im nahegelegenen Berlin sowie in überregionalen Strukturen der Flüchtlingshilfe ganz im Gegenteil die ,staatliche Umarmung“ und Indienstnahme sowie die allseitige Würdigung des Engagements im Zentrum der politischen Kritik (4). Mit dieser Ausrichtung von Voice wird das Engagement nicht (gegen Angriffe und Blockaden) stark gemacht, sondern seine Instrumentalisierung im Kontext (wohlfahrts-)staatlichen Versagens als Notlösung beanstandet und politisch auf seine Überwindung hingearbeitet. Die Büros für medizinische Flüchtlingshilfe - um nur ein Beispiel zu nennen - engagieren sich neben der ehrenamtlichen Gesundheitsversorgung von Geflüchteten politisch für eine öffentliche Gesundheitsversorgung für alle und problematisieren die staatliche Vereinnahmung ihres Engagements durch Auszeichnungen und Preise mit dem Slogan: „Es ist uns keine Ehre!“ (Schuh und Misbach 2015, S. 11) Diese Kritik geht davon aus, dass sich auch die beste Hilfe unter optimal geförderten Bedingungen als strukturell problematisch erweisen kann - und zwar überall dort, wo freiwilliges Engagement essenziell für die Gewährleistung grundlegender sozialer Leistungen und Infrastrukturen wird (s. a. van Dyk und Haubner 2019). Unter anderem wird hier auf das Problem einer ,philanthropic insufficiency“ (Salamon 1987, S. 39) hingewiesen, kann doch kein ehrenamtliches Angebot der Welt die immensen Kosten etwa für chronische Erkrankungen oder eine Krebsbehandlung tragen. Dieser Einwand wird v.a. von Akteuren erhoben, die bereits vor 2015 in den Bereichen Antirassismus, Flucht und Asyl aktiv waren und sich teilweise in langjährig gewachsenen Strukturen bewegen. Die Loyalität gegenüber Geflüchteten geht in diesen Fällen mit einer starken Selbstverpflichtung zu ihrer Unterstützung einher, wird zugleich aber mit einer Kritik staatlicher (Sozial-)Politik verbunden, die diese Solidarität ausbeutet.

Während der Verweis auf die Freiwilligkeit des Engagements also für einige - wie zuvor dargelegt - die Möglichkeit eines niedrigschwelligen Exits markiert, tritt bei anderen damit das Moment der Selbstverpflichtung in den Vordergrund, die selbst im Fall der kritischen Reflektion der Indienstnahme des eigenen Engagements einen Ausstieg verunmöglicht. Anders als bei der persönlichen Verantwortungsübernahme und Loyalität im heimlichen Engagement wird im zuletzt skizzierten Fall die Übernahme von Verantwortung nicht nur offen angemahnt, sondern auch zum Gegenstand einer ausdrücklichen politischen Positionierung. So betont Martin Kaiser vom Bun- 
desverband der Refugee Law Clinics: „Deshalb sehen wir uns in der Pflicht“ (Z. 195), ,wir haben vor allem das Gefühl, dass wir Verantwortung übernehmen müssen. Es fällt uns einfach wesentlich schwieriger zuzusehen, gerade wo wir wissen, dass wir etwas tun können. [...] Wir können auch helfen, dass Geflüchtete eine Stimme haben. Wir können Geflüchteten helfen, Zugang zum Recht zu bekommen. Und Zusehen war für uns keine Alternative.“ (Z. $312 \mathrm{ff}$.) Das Ziel, den Geflüchteten zu ihrem Recht zu verhelfen, ist hier mit der Hoffnung und dem Anliegen verbunden, ihnen selbst eine Stimme zu verleihen und die Möglichkeit zum Widerspruch und Protest zu verschaffen.

Mit dem Rückgriff auf die Trias Exit, Voice und Loyalty lassen sich also sowohl ein Ansatz zur Erklärung der hohen Abbruchquoten in diesem besonderen Engagementfeld gewinnen als auch die Ermöglichungsbedingungen unterschiedlicher Formen der Kritik herausarbeiten. Nicht zuletzt wird die ambivalente Rolle der Loyalität im Engagement für Geflüchtete ersichtlich, die einerseits dafür sorgt, dass Unverzichtbares (notdürftig) geleistet wird, damit andererseits aber potenziell eben jenes kritikwürdige staatliche Unterlassen kaschiert und legitimiert, das diese Leistung erst erforderlich gemacht hat.

\section{Fazit}

Unsere Forschungsergebnisse verdeutlichen, dass wir es bei der Flüchtlingshilfe mit einem Bereich des freiwilligen Engagements zu tun haben, der nach anderen Maßgaben funktioniert als die ehrenamtliche Unterstützung in anderen Feldern. Neben der Sondersituation des ,langen Sommers der Migration“ und der unisono konstatierten zwischenzeitlichen staatlichen Überlastung ist dies vor allem auf die besondere Vulnerabilität der Geflüchteten als Adressat*innen von Hilfe sowie die rassistisch und flüchtlingsfeindlich begründete Ablehnung des Engagements in Teilen der Gesellschaft zurückzuführen. Zudem ist die Flüchtlingshilfe heterogener als andere Engagementfelder, da unter diesem Titel höchst unterschiedliche Aufgabenbereiche versammelt sind, die im Blick auf staatliche Verantwortung und professionelle Standards sehr unterschiedliche Fragen aufwerfen - von der kurzfristigen Nothilfe durch die Versorgung mit Gütern des alltäglichen Bedarfs, über das langfristige Beziehungsengagement in Form von Betreuungen und Patenschaften, Sprachvermittlung und Übersetzungstätigkeiten, Freizeitangeboten, Kinderbetreuung und Unterstützung bei der Wohnungs- und Arbeitssuche bis hin zur medizinisch-therapeutischen Versorgung und Rechtsberatung. Hinzu kommt die Doppelrolle des Staates, der nicht nur für die Versorgung von Geflüchteten zuständig ist, sondern hoheitlich über Aufenthaltsstatus und Bleiberecht entscheidet, was wiederum zur Politisierung von Engagierten beigetragen hat.

Trotz dieser Spezifika macht die von uns untersuchte Unterstützung für Geflüchtete aber auch grundsätzliche Herausforderungen für die Engagementpolitik sowie für die Zukunft der sozialen Daseinsvorsorge wie unter einem Brennglas sichtbar. So sensibilisiert die große Engagement- und Hilfsbereitschaft in den Jahren 2015/2016 für die Bedeutung und den Wert kurzzeitiger, wenig institutionalisierter, häufig spontaner Unterstützung. Eine im Ministerium für Soziales und Integration 
Baden-Württemberg für Bürgerschaftliches Engagement zuständige Referentin betont, dass durch die Flüchtlingshilfe ,nochmal viel stärker deutlich [wurde], dass das kurzzeitige Engagement, das themenbezogene Engagement, das vorübergehende Engagement, Themen der Engagementpolitik sein müssen. Dass wir da neu draufgucken müssen. Das war ein sehr positiver Impuls." (Sonja Eger, Z. 546ff.)

Zusätzlich sensibilisiert unsere Studie für die spezifischen lokalen Kontexte und institutionellen Einbettungen des Engagements, zeigt sich doch, dass die Rahmenbedingungen des Engagements in Ost und West sowie im (groß-)städtischen und im ländlich/kleinstädtisch geprägten Raum diametral verschieden sind (Haubner et al. 2020): Während Engagierte in Groß- und Mittelstädten mit der staatlichen Indienstnahme ihres Engagements ringen und die rhetorische Würdigung ihrer Arbeit problematisieren, kämpfen viele in ländlich geprägten Regionen gegen Blockaden auf kommunaler Ebene und gegen alltägliche Anfeindungen von Geflüchteten und Engagierten. Wer über die Bedingungen des Engagements für Geflüchtete spricht, muss sich notwendig mit den Formen und Folgen von Alltagsrassismus auseinandersetzen.

Über die divergierenden Kontexte hinweg haben die prekären gesellschaftlichen Rahmenbedingungen der ehrenamtlichen Unterstützung für Geflüchtete derweil, so das zentrale Ergebnis unserer Analyse, multiple Grenzüberschreitungen zur Folge, die nur in ihrem Zusammenspiel begriffen werden können. Vier davon sind für uns einschlägig: die Überschreitung fachlicher und professioneller Grenzen durch nicht entsprechend qualifizierte Laien, politisch motivierte Grenzüberschreitungen (z.B. gegenüber Verwaltungsmitarbeiter*innen, die Ermessensspielräume nicht nutzen oder flüchtlingsfeindlich agieren), paternalistische Grenzüberschreitungen gegenüber Geflüchteten sowie das Überschreiten eigener (Belastungs-)Grenzen. Diese spezifischen Konstellationen sind jedoch nur dann in ihrer Verschränkung begreifbar, wenn die Rolle des Staates kritisch reflektiert und die freiwillig Engagierten nicht als „near-sacred figure[s]“ (Eliasoph 2013, S. 2) von vornherein jeglicher Kritik enthoben werden, wie es in Teilen des öffentlichen Diskurses zu beobachten ist.

Allerdings hat unsere Analyse auch gezeigt - und dieser Befund ist auch für andere Engagementbereiche von zentraler Bedeutung -, dass gerade die Freiwilligkeit als ein zentrales Charakteristikum und eine besondere Stärke des Engagements dazu beiträgt, dass strukturellen Problemen und Herausforderungen häufig nicht mit Kritik, Widerspruch und dem Willen zur Lösung, sondern mit Rückzug begegnet wird. Das gilt gleichwohl nicht immer, ist doch die Solidarität mit Geflüchteten und eine daraus erwachsende Selbstverpflichtung und Verantwortungsübernahme in vielen Fällen so stark ausgeprägt, dass das Engagement auch unter schwierigen Bedingungen - z.B. trotz empfundener Instrumentalisierung oder trotz alltäglicher Anfeindungen - fortgeführt wird. Während dabei das „,heimliche“ Engagement auf Sichtbarkeit verzichtet, verspricht ein seine Bedingungen öffentlich skandalisierendes Engagement die fruchtbarsten Anknüpfungspunkte für eine gegenstandsadäquate Engagement- und Gesellschaftspolitik: Eine solche würde die Unterstützung stärken, gleichzeitig aber auf die Kehrseiten und Probleme der Verzivilgesellschaftlichung der sozialen Frage aufmerksam machen. Von „Sternstunden der Zivilgesellschaft“ wäre in diesem Sinne dort zu sprechen, wo Engagierte der karitativen Engführung 
und Entpolitisierung der Zivilgesellschaft entgegentreten, ohne deshalb alltägliche Solidarität zu verwehren.

Danksagung Wir danken den anonymen Gutachter*innen des Berliner Journals für weiterführende Hinweise. Benjamin Seyd danken wir für sein aufmerksames Lektorat, von dem der vorliegende Artikel sehr profitiert hat.

Funding Open Access funding enabled and organized by Projekt DEAL.

Open Access Dieser Artikel wird unter der Creative Commons Namensnennung 4.0 International Lizenz veröffentlicht, welche die Nutzung, Vervielfältigung, Bearbeitung, Verbreitung und Wiedergabe in jeglichem Medium und Format erlaubt, sofern Sie den/die ursprünglichen Autor(en) und die Quelle ordnungsgemäß nennen, einen Link zur Creative Commons Lizenz beifügen und angeben, ob Änderungen vorgenommen wurden.

Die in diesem Artikel enthaltenen Bilder und sonstiges Drittmaterial unterliegen ebenfalls der genannten Creative Commons Lizenz, sofern sich aus der Abbildungslegende nichts anderes ergibt. Sofern das betreffende Material nicht unter der genannten Creative Commons Lizenz steht und die betreffende Handlung nicht nach gesetzlichen Vorschriften erlaubt ist, ist für die oben aufgeführten Weiterverwendungen des Materials die Einwilligung des jeweiligen Rechteinhabers einzuholen.

Weitere Details zur Lizenz entnehmen Sie bitte der Lizenzinformation auf http://creativecommons.org/ licenses/by/4.0/deed.de.

\section{Literatur}

Amann, M. et al. (2015). Das neue Deutschland. Der Spiegel vom 28.08.2015. URL: https://www.spiegel. de/politik/das-neue-deutschland-a-19b5ca08-0002-0001-0000-000138379331?context=issue. Zugegriffen: Aug. 2021.

Anonym (2015). Gewalt gegen ehrenamtliche Helfer. Märkische Allgemeine vom 11.11.2015 (S. 7).

AWO Bundesverband e.V. (2015). Freiwilliges Engagement in der AWO Flüchtlingsarbeit. Eine Arbeitshilfe und Ideensammlung. Berlin.

BAFzA (Bundesamt für Familie und zivilgesellschaftliche Aufgaben) (2015). Merkblatt zum Sonderprogramm Bundesfreiwilligendienst mit Flüchtlingsbezug. Berlin.

Bagfa (Bundesarbeitsgemeinschaft der Freiwilligenagenturen) (2016). Im Spiegel der Freiwilligenagenturen: Das Engagement für und mit Flüchtlingen. Herausforderungen und Gestaltungsmöglichkeiten. Berlin.

Beckmann, F., Hoose, F., \& Schönauer, A.-L. (2018). „Wir hatten bis jetzt auch erstaunlich wenig mit Nazis zu tun". Gesellschaftliche Stimmungen und ihre Wahrnehmung in der Flüchtlingshilfe. In S. Zajak \& I. Gottschalk (Hrsg.), Flüchtlingshilfe als neues Engagementfeld (S. 23-52). Baden-Baden: Nomos.

Bertelsmann Stiftung (Hrsg.). (2016). Koordinationsmodelle und Herausforderungen ehrenamtlicher Flüchtlingshilfe in den Kommunen. Qualitative Studie des Berliner Instituts für empirische Integrations- und Migrationsforschung. Berlin: BIM.

BMFSFJ (Bundesministerium für Familie, Senioren, Frauen und Jugend) (2016). Zweiter Engagementbericht: Demografischer Wandel und bürgerschaftliches Engagement zur lokalen Entwicklung. Berlin: BMFSFJ.

BMFSFJ (Bundesministerium für Familie, Senioren, Frauen und Jugend) (2017). Engagement in der Flüchtlingshilfe. Ergebnisbericht einer Untersuchung des Instituts für Demoskopie Allensbach. Berlin: BMFSFJ. URL: https://www.bmfsfj.de/blob/122010/d35ec9bf4a940ea49283485db4625aaf/ engagement-in-der-fluechlingshilfe-data.pdf. Zugegriffen: Aug. 2021.

BMI (Bundesministerium des Innern, für Bau und Heimat) (2018). Kooperation von Haupt- und Ehrenamtlichen in der Arbeit mit Geflüchteten. Frankfurt a. M.: BMI.

Boemke, L., van Dyk, S., \& Haubner, T. (2021). Freiwilligenarbeit als Ressource. Die Indienstnahme von Engagement und die subjektiven Perspektiven der Engagierten. WSI-Mitteilungen, 74, 374-384.

Bohnsack, R. (2003). Rekonstruktive Sozialforschung. Einführung in qualitative Methoden. Opladen: Leske + Budrich. 
Braun, K. (2017). Decolonial perspectives on charitable spaces of „Welcome Culture“ in Germany. Social Inclusion, 5(3), 38-48.

Breining, T. (2015). Das DRK sieht keine Spielräume mehr. Stuttgarter Zeitung vom 06.10.2015.

Buscham, L., \& Gillwald, S. (2018). OpenTransfer \# Patenschaften. In BBE (Hrsg.), Newsletter für Engagement und Partizipation in Deutschland 2/2018. URL: https://www.b-b-e.de/fileadmin/Redaktion/ 05_Newsletter/01_BBE_Newsletter/2018/newsletter-02-buscham-gillwald.pdf. Zugegriffen: Aug. 2021.

Byakuleka, B.B., \& Ulu, T. (2016). Der Rassismus des Helfens. Warum so viele Willkommensinitiativen nicht politisch über die Flüchtlingsfrage sprechen wollen. Analyse und Kritik, (613), 18.

Dahme, H.-J., \& Wohlfahrt, N. (2009). Zivilgesellschaft und „managerieller“ Staat. In I. Bode, A. Evers \& A. Klein (Hrsg.), Bürgergesellschaft als Projekt (S. 240-264). Wiesbaden: VS Verlag für Sozialwissenschaften.

Deggerich, M., Neumann, C., \& Popp, M. (2015). Vor dem Zusammenbruch. Der Spiegel vom 31.10.2015.

Denninger, T., van Dyk, S., Lessenich, S., \& Richter, A. (2014). Leben im Ruhestand. Zur Neuverhandlung des Alters in der Aktivgesellschaft. Bielefeld: transcript.

Der Paritätische (2014). Ehrenamt - Freiwilliges soziales Engagement im Paritätischen Berlin. Paritätischer Rundbrief, August/September, 32-34. URL: https://www.paritaet-berlin.de/fileadmin/user_ upload/Dokumente/Rundbriefe/2014-08-09_PAR_Rundbrief_web.pdf. Zugegriffen: Aug. 2021.

Deutscher Bundestag (2012). Entwurf und Begründung eines Gesetzes zur Entbürokratisierung des Gemeinnützigkeitsrechts. Drucksache 17/11316. Berlin.

Deutscher Bundestag (2017). Zweiter Bericht über die Entwicklung des bürgerschaftlichen Engagements in der Bundesrepublik Deutschland. Drucksache 18/11800. Berlin.

Diemand, S. (2015). Nur gut gemeint reicht nicht aus. die tageszeitung vom 05.09.2015.

van Dyk, S. (2019). Von der Nothilfe zur politischen Ökonomie des Helfens. In K. Binner \& K. Scherschel (Hrsg.), Fluchtmigration und Gesellschaft (S. 32-49). Weinheim: Beltz Juventa.

van Dyk, S., \& Haubner, T. (2019). Gemeinschaft als Ressource? Engagement und Freiwilligenarbeit im Strukturwandel des Wohlfahrtsstaats. In A.D. Baumgartner \& B. Fux (Hrsg.), Sozialstaat unter Zugzwang? (S. 259-280). Wiesbaden: Springer VS.

van Dyk, S., \& Haubner, T. (2021). Community-Kapitalismus. Hamburg: Hamburger Edition.

van Dyk, S., Boemke, L., \& Haubner, T. (2020). Fallstricke des Helfens oder Sternstunden der Solidarität? Engagement für Geflüchtete im Spannungsfeld von Indienstnahme, Rassismus, Charity und Politisierung. Bürger und Staat, 70(3), 136-143.

Eliasoph, N. (2013). The politics of volunteering. Cambridge: Polity Press.

Fassin, D. (2012). Humanitarian reason: A moral history of the present. Berkeley: University of California Press.

Flüchtlingsrat Brandenburg (2015). Offener Brief von flüchtlingspolitischen Initiativen/Willkommensinitiativen im Land Brandenburg. URL: https://www.fluechtlingsrat-brandenburg.de/wp-content/ uploads/2015/12/14_12_2015_Offener_Brief_Initiativen.pdf. Zugegriffen: Aug. 2021.

Gebauer, T. (2016). Hilfe - Politik - Solidarität. Ein Plädoyer für Reflexion. URL: https:// geschichtedergegenwart.ch/hilfe-politik-solidaritaet-ein-plaedoyer-fuer-reflexion/. Zugegriffen: Aug. 2021.

Gesemann, F., \& Roth, R. (2017). Erfolgsfaktoren kommunaler Integration von Geflüchteten. Berlin: Friedrich-Ebert-Stiftung.

Graf, L. (2016). Freiwillig im Ausnahmezustand: Die ambivalente Rolle ehrenamtlichen Engagements in der Transformation des Asylregimes. Widersprüche, 36(141), 87-96.

Hamann, U., Karakayali, S., Wallis, M., \& Höfler, L. (2016). Erhebung zu Koordinationsmodellen und Herausforderungen ehrenamtlicher Flüchtlingshilfe in den Kommunen. Gütersloh: Bertelsmann Stiftung.

Haubner, T. (2017). Die Ausbeutung der sorgenden Gemeinschaft. Laienpflege in Deutschland. Frankfurt a. M.: Campus.

Haubner, T., van Dyk, S., \& Boemke, L. (2020). „Im Westen nichts Neues, im Osten noch selten“? Freiwilliges Engagement im Spannungsfeld von Nachwende-Erbe und neuen Herausforderungen. Zeitschrift für Freiwilligendienste, 1, 54-69.

Hess, Sabine et al. (Hrsg.). (2017). Der Lange Sommer der Migration. Grenzregime II. Berlin: Assoziation A.

Hirschman, A. O. (1970). Exit, Voice and Loyalty. Responses to Decline in Firms, Organizations and States. Cambridge: Harvard University Press.

Jakob, C. (2015). Das eigene Sofa einem Flüchtling anzubieten, ist wichtig. Noch wichtiger ist aber, dass Flüchtlinge selbst entscheiden, was sie wollen. die tageszeitung vom 15.08.2015. 
Joffe, J. (2015). Das deutsche Wunder. Zeit-online vom 10.09.2015. URL: https://www.zeit.de/2015/37/ willkommenskultur-deutschland-fluechtlinge-zeitgeist. Zugegriffen: Aug. 2021.

Jungk, S. (2016). Willkommenskultur: Von neuen Chancen, alten Fehlern und Versäumnissen. Widersprüche, 36(3), 99-108.

Jürgens, K. (2010). Deutschland in der Reproduktionskrise. Leviathan, 38, 559-587.

Karakayali, S. (2017). Feeling the scope of solidarity: The role of emotions for volunteers supporting refugees in Germany. Social Inclusion, 5(3), 7-16.

Karakayali, S., \& Kleist, O. (2015). Strukturen und Motive der ehrenamtlichen Flüchtlingsarbeit in Deutschland. Eine Studie des Berliner Instituts für empirische Integrations- und Migrationsforschung. Berlin: BIM.

Kelnberger, J. (2015). Hass, Hilfe, Hoffnung. Süddeutsche Zeitung vom 20.07.2015.

Kessl, F., \& Wagner, T. (2011). „Was vom Tisch der Reichen fällt...“: zur neuen politischen Ökonomie des Mitleids. Widersprüche, 31(119/120), 55-76.

Klein, A. (2016). Der Eigensinn des Engagements als Voraussetzung guter Engagementpolitik. Forschungsjournal Soziale Bewegungen, 28(1), 144-149.

Kleist, O. J. (2018). Flucht- und Flüchtlingsforschung in Deutschland: Akteure, Themen und Strukturen. State-of-Research Papier 01. IMIS: Osnabrück.

Kleist, O. J. (2017). „Das große Engagement in der ehrenamtlichen Flüchtlingsarbeit zeigt: Die Zivilgesellschaft ist offen für Migration.“ Ein Interview der Bundeszentrale für Politische Bildung. URL: https://www.bpb.de/gesellschaft/migration/kurzdossiers/250255/interview-mit-j-olaf-kleist. Zugegriffen: Aug. 2021.

Lessenich, S. (2008). Die Neuerfindung des Sozialen. Der Sozialstaat im flexiblen Kapitalismus. Bielefeld: transcript.

Memarnia, S. (2015). „Wir sollten uns nicht wundern, dass sie kommen“. die tageszeitung vom 15.10.2015.

Ministerium für Arbeit, Soziales, Gesundheit, Frauen und Familie Brandenburg (2018). „Integration machen Menschen". Aktuelle Situation des Ehrenamts in der Flüchtlingshilfe im Land Brandenburg. Potsdam: MASGF. URL: https://msgiv.brandenburg.de/sixcms/media.php/9/web-13032018Kurzfassung-Ehrenamt.pdf. Zugegriffen: Aug. 2021.

Müller, C.-P. (2016). Erst der Körper, dann die Seele. Frankfurter Allgemeine Zeitung vom 23.03.2016.

Mutz, G., \& Wolff, L. (2018). Besonderheiten des freiwilligen Engagements für geflüchtete Menschen. Empirische Befunde und sozialwissenschaftliche Verortung. In S. Zajak \& I. Gottschalk (Hrsg.), Flüchtlingshilfe als neues Engagementfeld (S. 53-76). Baden-Baden: Nomos.

Mutz, G., et al. (2015). Engagement für Flüchtlinge in München. Ergebnisse eines Forschungsprojekts an der Hochschule München in Kooperation mit dem Münchner Forschungsinstitut miss. München. URL: https://w3-mediapool.hm.edu/mediapool/media/dachmarke/dm_lokal/presse/pm/2015_ 4/Abschlussbericht_final_30092015.pdf. Zugegriffen: Aug. 2021.

Neumann, D. (2016). Das Ehrenamt nutzen. Zur Entstehung einer staatlichen Engagementpolitik in Deutschland. Bielefeld: transcript.

Petersen, M. (2018). Ehrenamtliche Dolmetscher im Gesundheitsbereich - sinnvoll oder nicht? In BBE (Hrsg.), Newsletter für Engagement und Partizipation in Deutschland 1/2018. URL: https://www. b-b-e.de/fileadmin/Redaktion/05_Newsletter/01_BBE_Newsletter/2018/newsletter-01-petersen.pdf. Zugegriffen: Aug. 2021.

Phineo (2016). Vom Willkommen zum Ankommen. Ratgeber für wirksames Engagement für Flüchtlinge in Deutschland. Berlin: Phineo.

Pinl, C. (2010). Solidaritätsdumping. die tageszeitung vom 19.10.2010.

Rose, N. (2000). Tod des Sozialen? Eine Neubestimmung der Grenzen des Regierens. In U. Bröckling, S. Krasmann \& T. Lemke (Hrsg.), Gouvernementalität der Gegenwart (S. 72-109). Frankfurt a. M.: Suhrkamp.

Roth, R. (2003). Die dunklen Seiten der Zivilgesellschaft. Forschungsjournal Neue Soziale Bewegungen, 16(2), 59-73.

Salamon, L.M. (1987). Of market failure, voluntary failure, and third-party government: Toward a theory of government-nonprofit relations in the modern welfare state. Nonprofit and Voluntary Sector Quarterly, 16, 29-49.

Schäuble, B. (2017). Zwischen Institution und Community. Freiwillige in den Hilfen zur Erziehung. In B. Schäuble \& L. Wagner (Hrsg.), Partizipative Hilfeplanung (S. 127-140). Weinheim: Beltz Juventa.

Schuh, H., \& Misbach, E. (2015). Es ist uns keine Ehre. In apabiz \& MBR (Hrsg.), Berliner Zustände 2014. Ein Schattenbericht über Rechtsextremismus, Rassismus und Antisemitismus im Jahr 2014 (S. 12-17). Berlin. URL: https://www.apabiz.de/wp-content/uploads/2014schattenbericht-web.pdf. Zugegriffen: Aug. 2021. 
Selke, S. (Hrsg.). (2009). Tafeln in Deutschland. Aspekte einer sozialen Bewegung zwischen Nahrungsmittelumverteilung und Armutsintervention. Wiesbaden: VS Verlag für Sozialwissenschaften.

Simonson, J., \& Vogel, C. (2020). Freiwilliges Engagement im Alter. In K. R. Schroeter, H. Künemund \& C. Vogel (Hrsg.), Handbuch Soziologie des Alter(n)s (S. 1-31). Wiesbaden: Springer VS.

Speth, R., \& Becker, E. (2016). Zivilgesellschaftliche Akteure und die Betreuung geflüchteter Menschen in deutschen Kommunen. Maecenata Institut, Opuscolum Nr. 92. Berlin. URL: https://repository.difu. de/jspui/bitstream/difu/226321/1/DM16041359.pdf. Zugegriffen: Aug. 2021.

Staatsministerium Baden-Württemberg (2015). Willkommen! Ein Handbuch für die ehrenamtliche Flüchtlingshilfe in Baden-Württemberg. Stuttgart.

Stadler, S. (2016). Hilfen für Helfer. Fünf Handlungsempfehlungen an die Bundesregierung. In BBE (Hrsg.), Newsletter für Engagement und Partizipation in Deutschland 9/2016. URL: https://www. b-b-e.de/fileadmin/Redaktion/05_Newsletter/01_BBE_Newsletter/bis_2017/2016/newsletter-09. stadler.pdf. Zugegriffen: Aug. 2021.

Steinhilper, E. (2017). Politisiert in der Migration, vernetzt in der Stadt. Transnationaler politischer Protest von Geflüchteten in Berlin. Forschungsjournal Soziale Bewegungen, 30(3), 77-87.

Strauss, A. L. (2004). Methodologische Grundlagen der Grounded Theory. In J. Strübing \& B. Schnettler (Hrsg.), Methodologie interpretativer Sozialforschung. Klassische Grundlagentexte (S. 427-451). Konstanz: UVK.

Vey, J., \& Sauer, M. (2016). Ehrenamtliche Flüchtlingsarbeit in Brandenburg. Hrsg. vom Aktionsbündnis Brandenburg. Berlin: Institut für Protest- und Bewegungsforschung.

Wagenknecht, S. (2016). Hilfe für die Helfer. In BBE (Hrsg.), Newsletter für Engagement und Partizipation in Deutschland 9/2016. URL: https://www.b-b-e.de/fileadmin/Redaktion/05_Newsletter/01_ BBE_Newsletter/bis_2017/2016/newsletter-09-wagenknecht.pdf. Zugegriffen: Aug. 2021.

Zeckra, C. (2017). Netzwerkst Du noch oder kooperierst Du schon? In BBE (Hrsg.), Newsletter für Engagement und Partizipation in Deutschland 8/2017. URL: https://www.lokale-demokratie.de/wpcontent/uploads/2017/04/newsletter-08-zeckra-integration.pdf. Zugegriffen: Aug. 2021.

Silke van Dyk geb. 1972. Dr. rer. soc., Professorin für Politische Soziologie an der Friedrich-SchillerUniversität Jena. Forschungsschwerpunkte: Politische Soziologie, Soziologie der Sozialpolitik und des Wohlfahrtsstaats, Soziologie des Alters und der Demografie, Gesellschafts- und Demokratietheorie, Diskurstheorie und empirische Diskursforschung, qualitative Sozialforschung. Aktuelle Veröffentlichungen: (mit T. Haubner) Community-Kapitalismus, 2021; (mit F. Kessel, Hrsg.) Freiwilligenarbeit im Strukturwandel des Wohlfahrtsstaats. Schwerpunktheft der WSI-Mitteilungen, 2021; Solidarität revisited. Die soziale Frage, die Wiederentdeckung der Gemeinschaft und der Rechtspopulismus, in: L. Susemichel \& J. Kastner (Hrsg.), Unbedingte Solidarität, 2021.

Laura Boemke geb. 1989. M. A., wissenschaftliche Mitarbeiterin am Institut für Soziologie der FriedrichSchiller-Universität Jena. Forschungsschwerpunkte: Soziologie der Sozial- und Arbeitsmarktpolitik sowie des Wohlfahrtsstaats, informelle Ökonomien, Erwerbslosigkeit, Armut, soziale Ungleichheit und regionale Deprivation, Engagementforschung. Aktuelle Veröffentlichungen: (mit S. van Dyk und T. Haubner) Freiwilligenarbeit als Ressource. Die Indienstnahme von Engagement und die subjektiven Perspektiven der Engagierten, in: S. van Dyk \& F. Kessl (Hrsg.), Freiwilligenarbeit im Strukturwandel des Wohlfahrtsstaats. Schwerpunktheft der WSI-Mitteilungen, 2021.

Tine Haubner geb. 1983. Dr. phil., wissenschaftliche Mitarbeiterin am Institut für Soziologie der Friedrich-Schiller-Universität Jena. Forschungsschwerpunkte: Ränder der bundesrepublikanischen Arbeitsgesellschaft, soziale Reproduktion und Care, Sozialpolitik und Wohlfahrtsstaat, qualitative Methoden der Sozialforschung. Aktuelle Veröffentlichungen: (mit S. van Dyk) Community-Kapitalismus, 2021; „Da könnte es ja auch ein weniger Ausgebildeter machen." Freiwilligenarbeit im Spannungsfeld umkämpfter Professionalisierung in den Sozialberufen, in: S. van Dyk \& F. Kessl (Hrsg.), Freiwilligenarbeit im Strukturwandel des Wohlfahrtsstaats. Schwerpunktheft der WSI-Mitteilungen, 2021; (mit S. van Dyk und L. Boemke) Gemeinwohldienst oder Gratisarbeit? Zur politischen Ökonomie von Freiwilligenarbeit im Gegenwartskapitalismus, in: Prokla, 2021. 